Mesopotamia J. of Agric.

Vol. (45) No. (4) 2017

التدهور الوراثي والتحسين الوراثي المتوقع لهجن الجيل الثاني في الباقلاء (Vicia faba L)

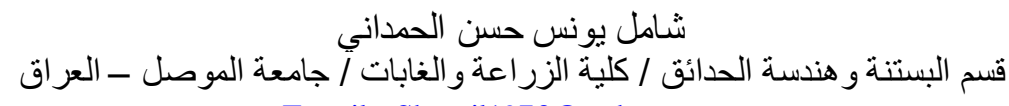 \\ E-mail:Shamil1970@yahoo.com
}

\begin{abstract}
الخلاصة فزية
\end{abstract}

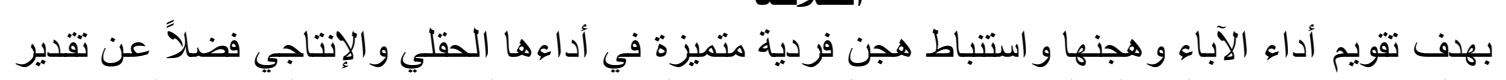

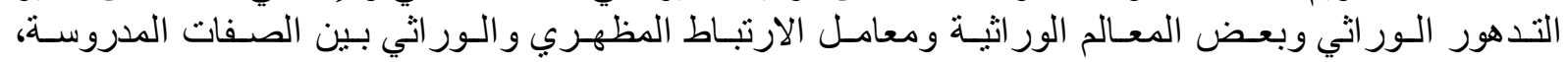

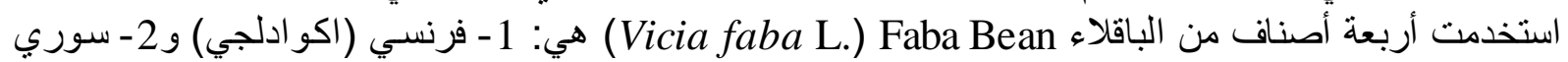

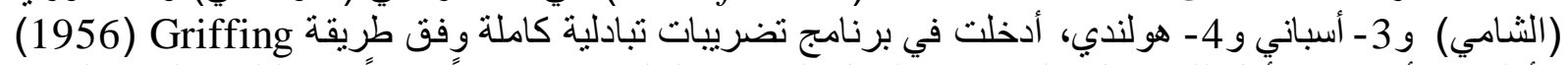

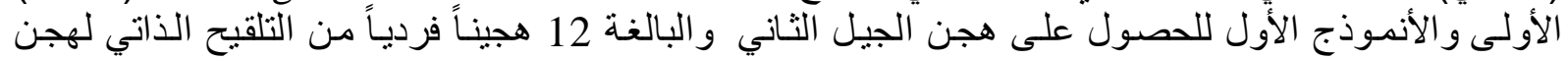

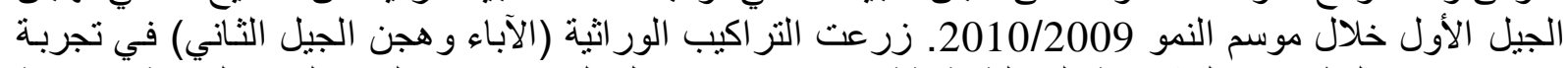

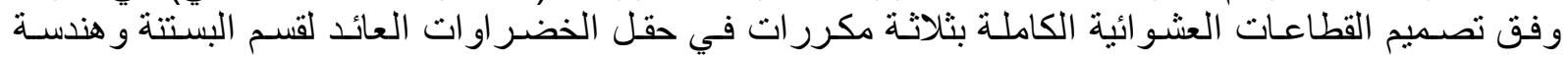

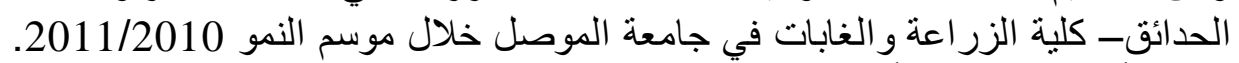

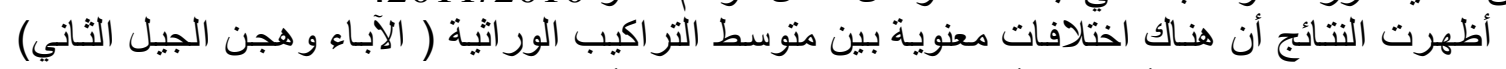

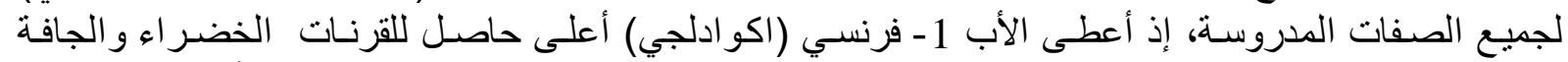

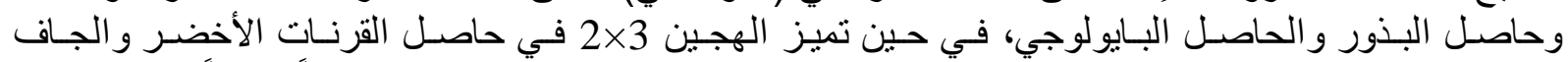

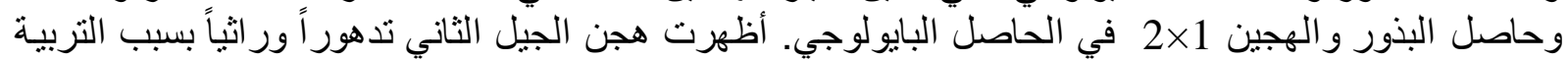

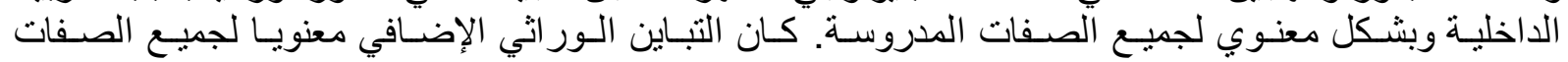

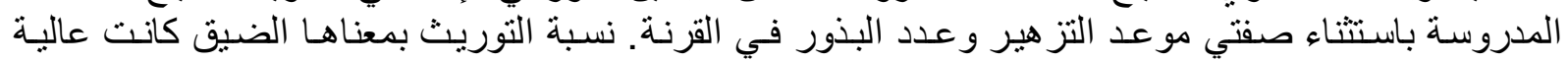

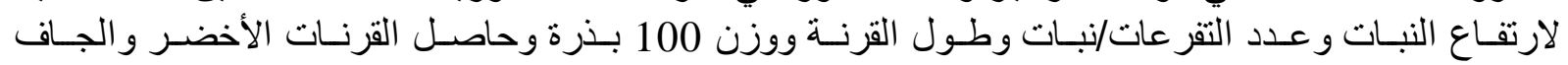

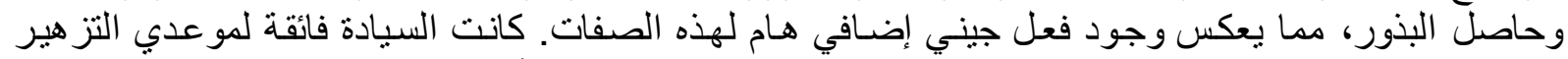

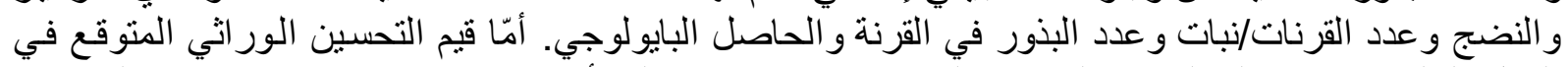

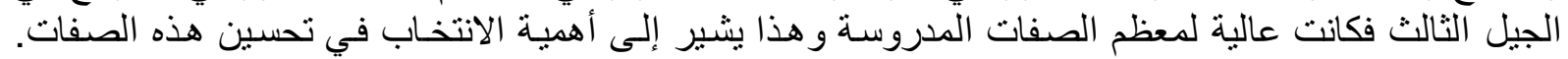

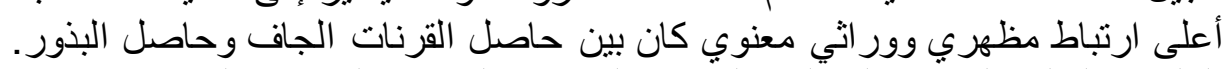

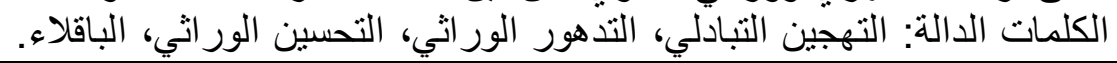

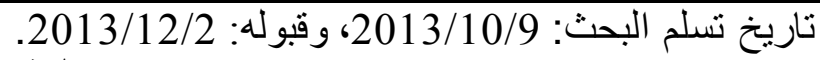 المقدمة
}

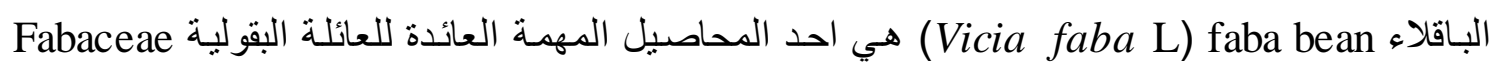

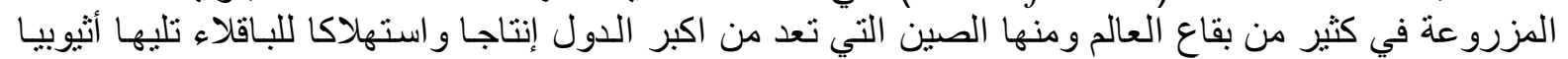

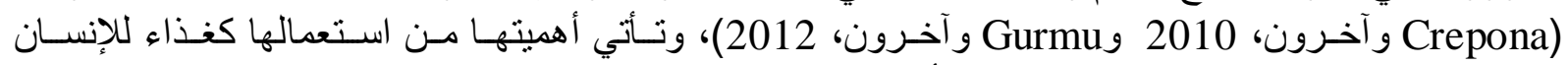

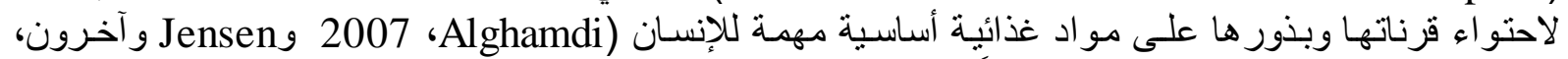

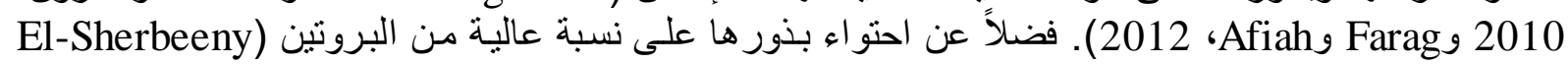

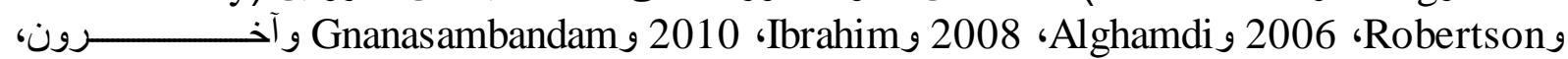

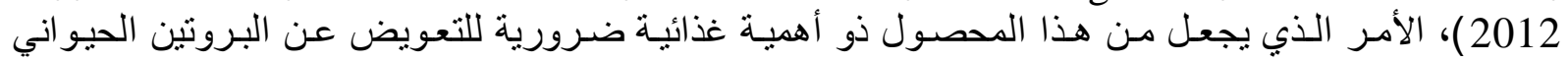

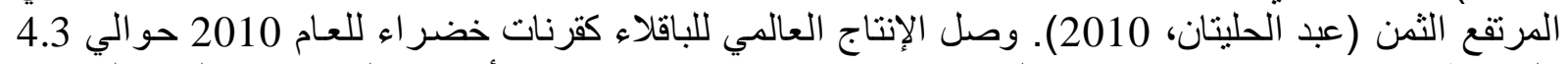

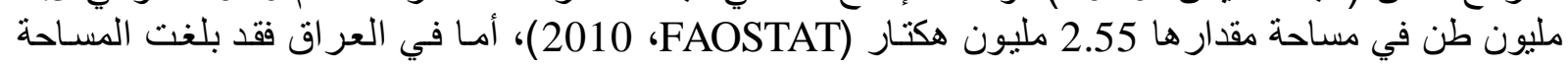

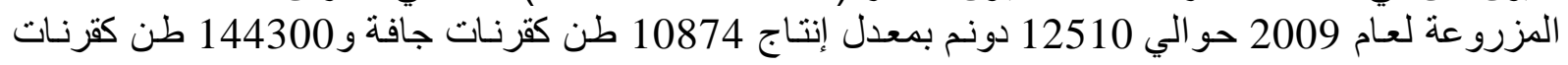

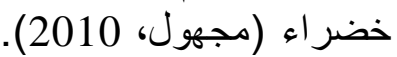
إن دراسة التربية الداخلية في ور اثثة الصفات الكمية ينصب في اتجاهين الأول هو تأثثير ها على معدل أداء

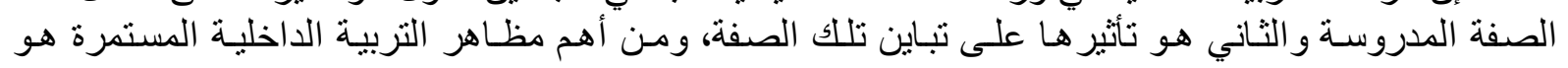

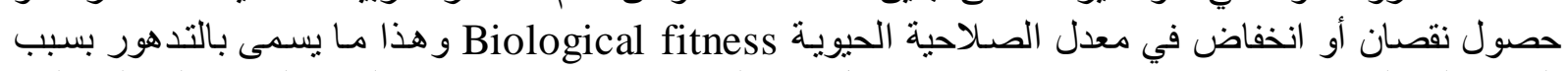

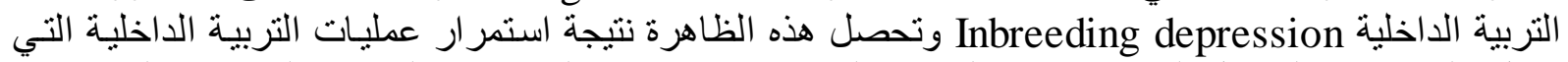

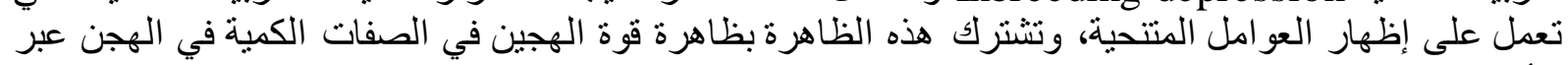

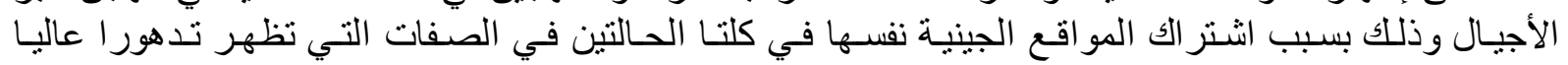

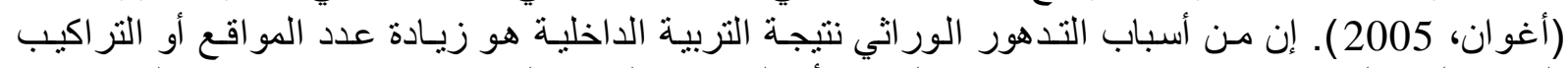

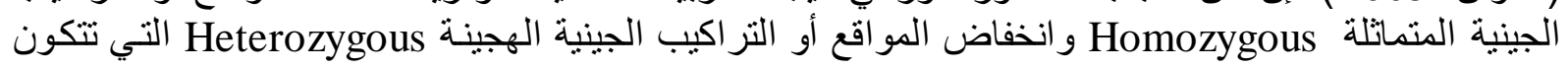


Mesopotamia J. of Agric.

Vol. (45) No. (4) 2017
ISSN: $2224-9796$ (Online)

ISSN: 1815 - $316 \mathrm{X}$ (Print)

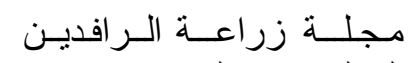

المجلد (45) العدد (4) 2017

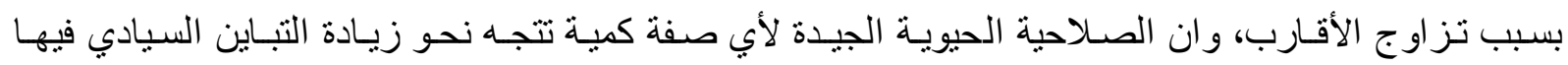

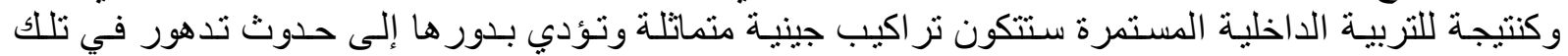
الصفة. وجد Abdalla و آخـرون (2001) و و (2012 و آخرون (2003) و

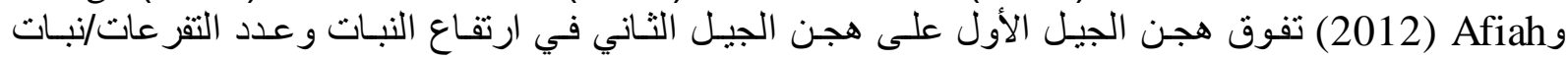

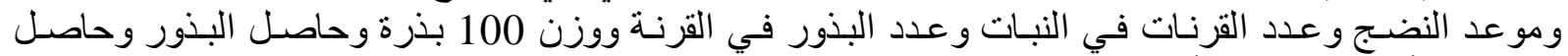

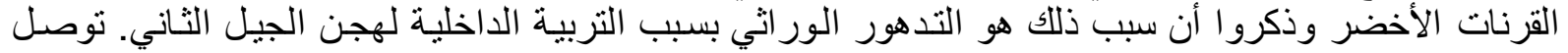

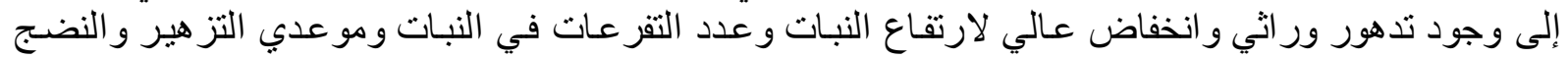

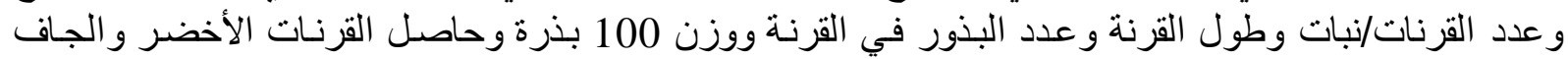

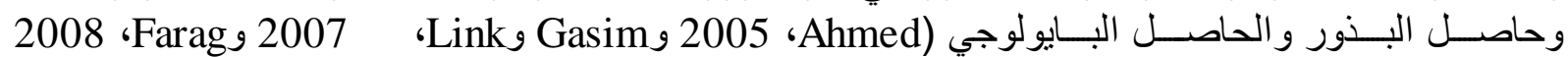

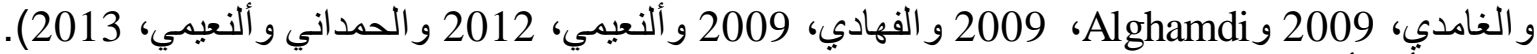

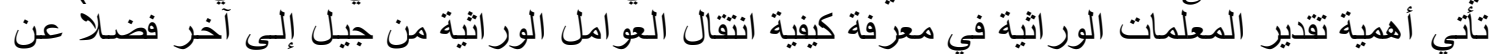

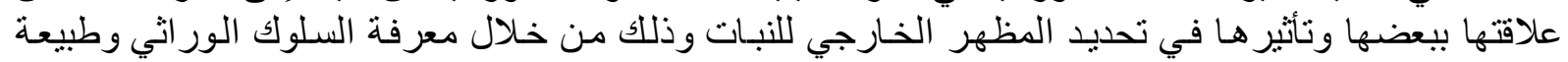

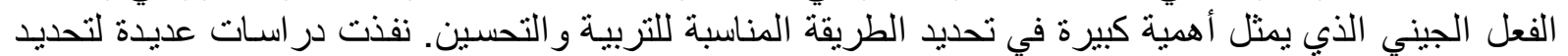

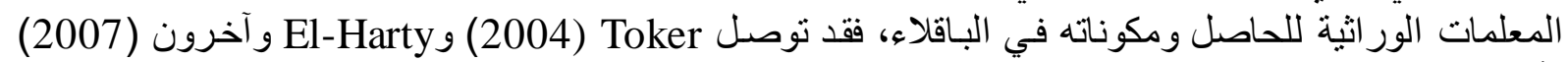

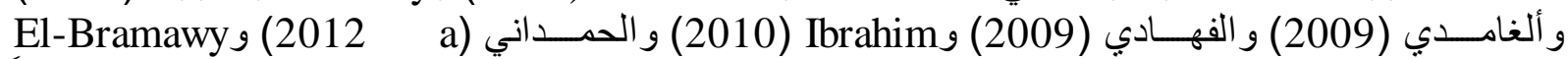

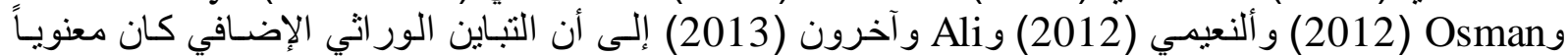

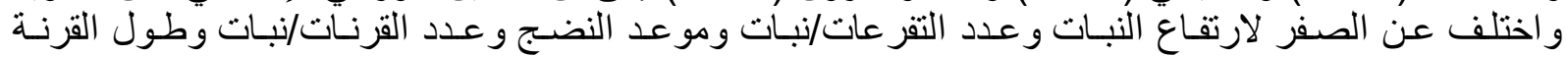

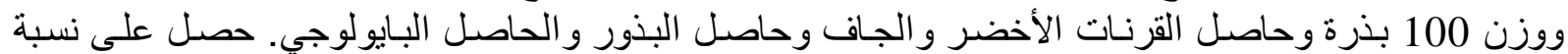

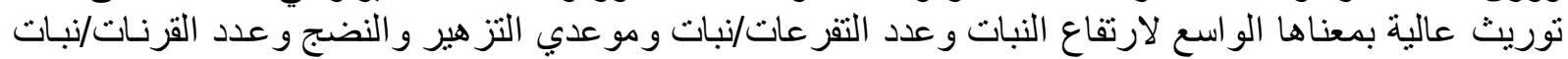

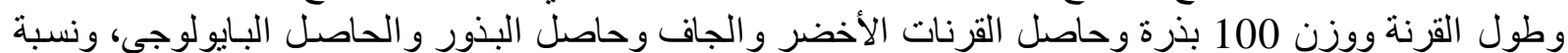

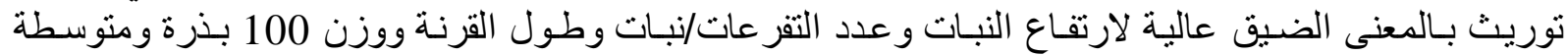

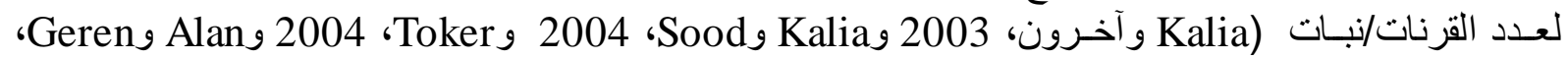

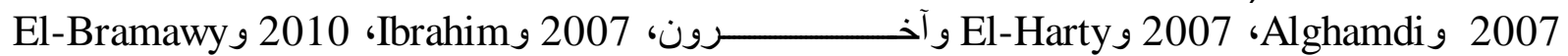

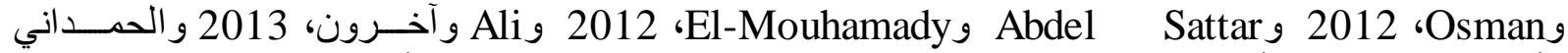

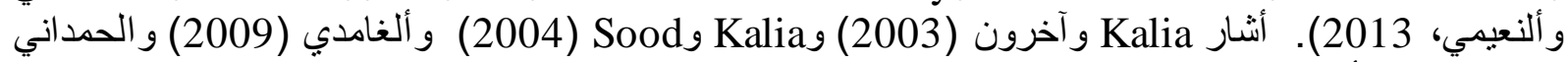

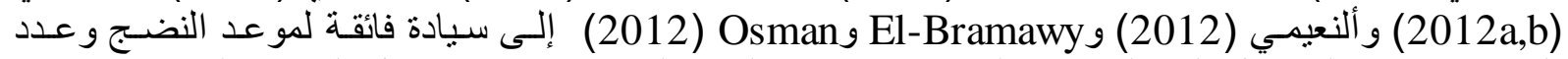

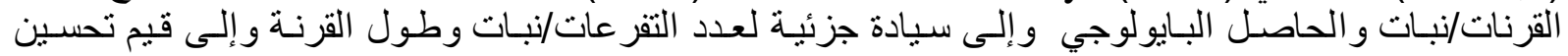

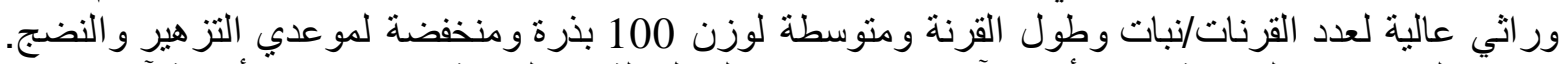

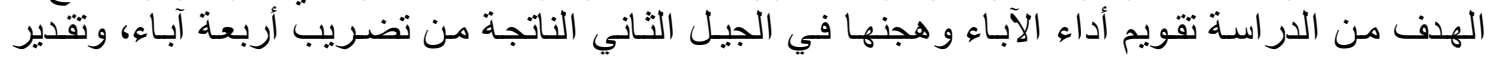

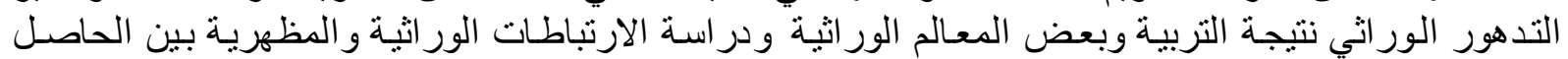

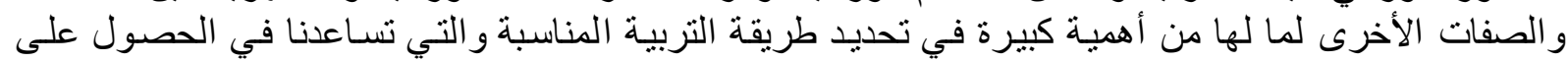

\section{مواد البحث وطر ائقه}

الصنف المرغوب.

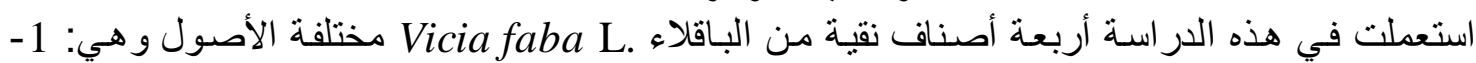

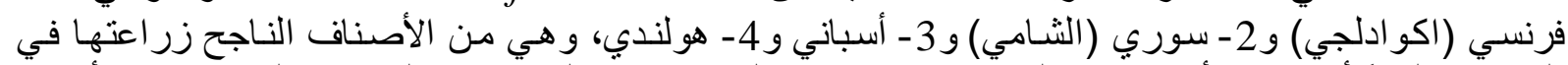

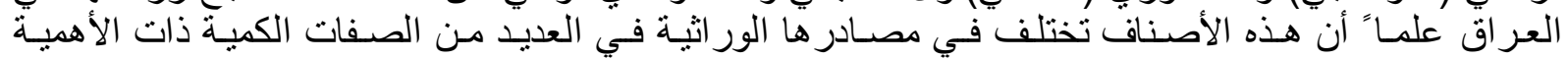

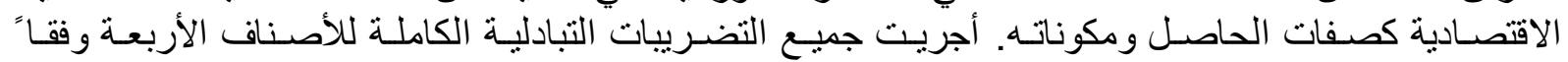

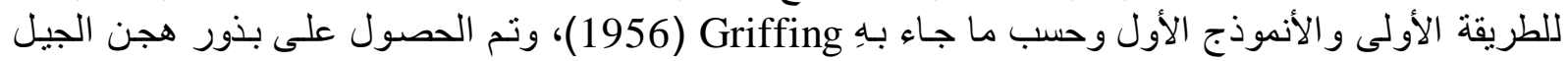

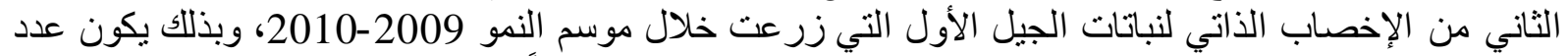

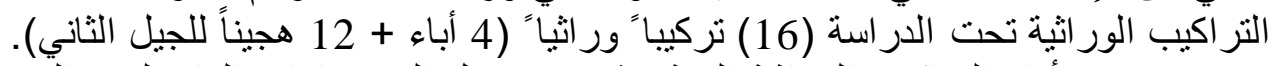

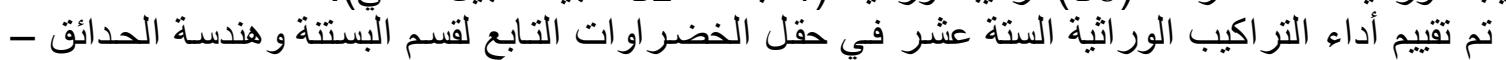

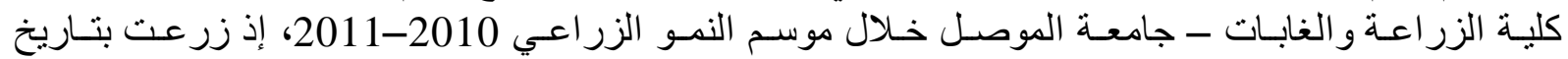

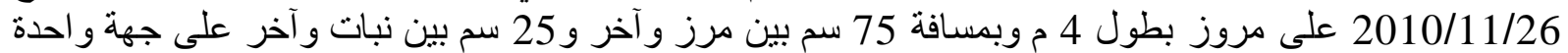

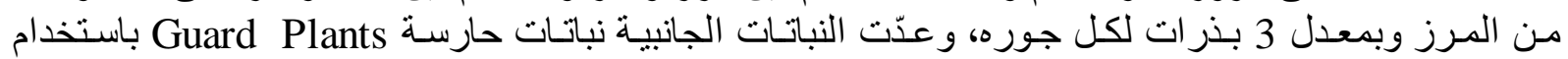

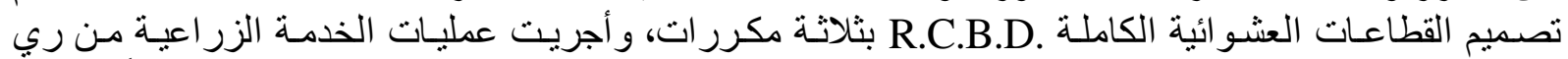

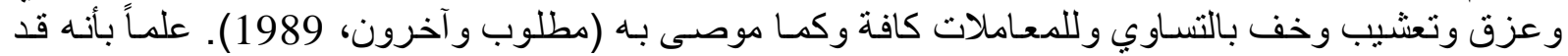
أجريت عملية الخف بحيث تحوي كل جوره على نبات واحد (المعيوف، 1982). تم مكافحة الأدغال يدوياً أثناء 
Mesopotamia J. of Agric.

Vol. (45) No. (4) 2017
ISSN: $2224-9796$ (Online)

ISSN: 1815 - $316 \mathrm{X}$ (Print)

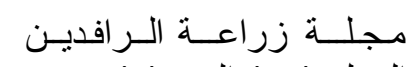

المجلد (45) العدد (4) 2017

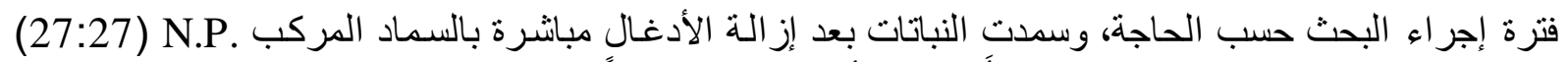

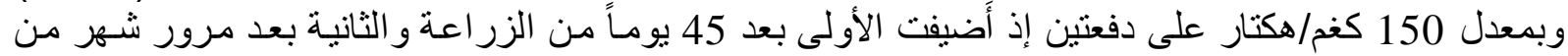

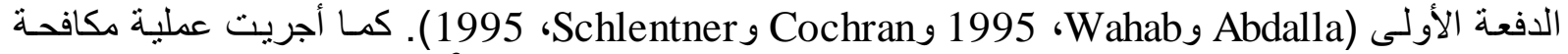

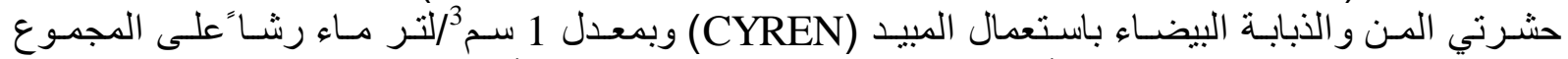

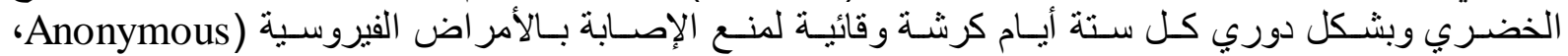

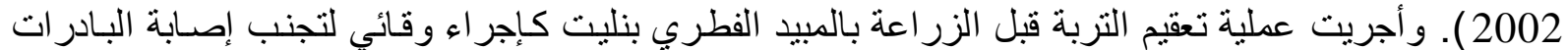
بالأمر اض الفطرية.

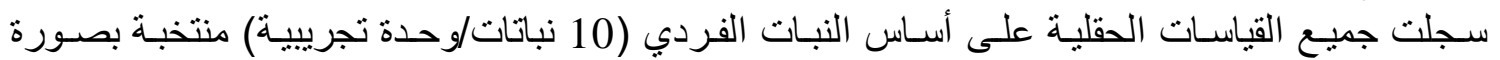

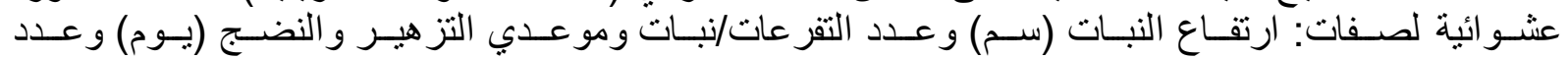

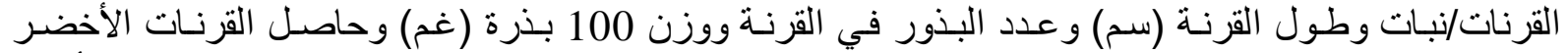

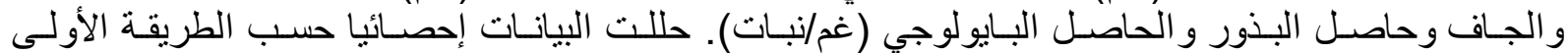

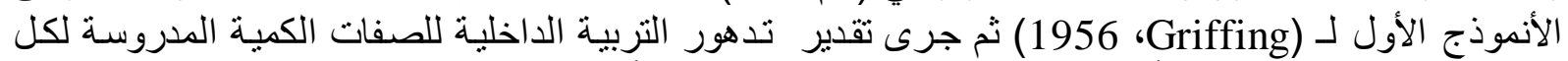

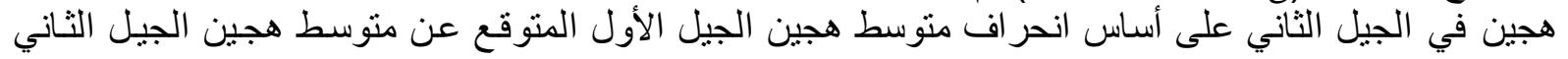

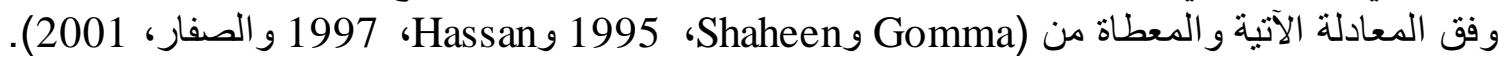

$$
I=E \overline{F_{1}}-O \overline{F_{2}}
$$

= I S منوسط هجين الجيل الأول المنوقع. = Oت متوسط هجين الجيل الثاني المشاهد. إذ تم حسـاب متوسط هجين الجيل الأول المتوقع باعتمـاد المعادلة المقدمة من قبـل Mather و Jinks

$$
\begin{aligned}
& E \bar{F} 1=2 \bar{F} 2-(1 / 2) \bar{P} 1-(1 / 2) \bar{P} 2 \\
& \text { E متوسط هجين الجيل الأول المنوقع. } \\
& \text { = F } 2 \\
& \text { متوسط الصنف الأبوي الأول (من معدل المشاهدة). } \\
& \text { الصنو الصنف الأبوي الثاني (من معدل المشاهدة) }
\end{aligned}
$$

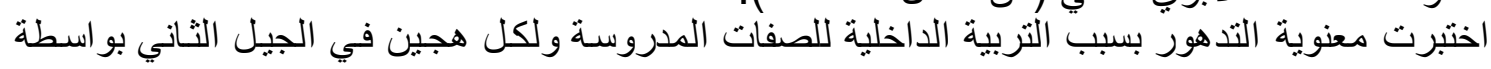

اختبار t وقدرت قيمة t بالمعادلة الآتية:

$$
t=\frac{I}{\sqrt{V(I)}}
$$

\section{حيث أن:}

=I

SEI = $\sqrt{V(I)}$ وحسب تباين التدهور الداخلية V(I) لكل صفة مدروسة بالمعادلة الآتية:

$$
V(I)=V \overline{F_{1}}-V \bar{F}_{2}
$$

إذ أن:

= V (I)

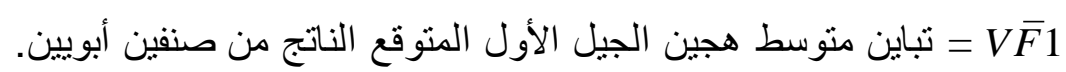

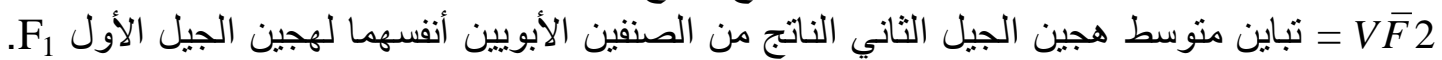

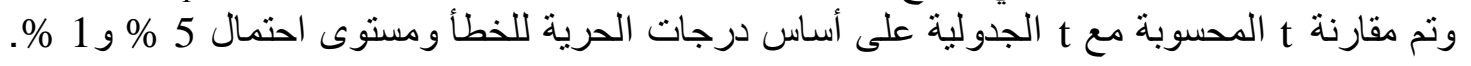
قدر التباين الور اثي الإضافي $\sigma_{A}^{2}$ و السيادي EMS 
Mesopotamia J. of Agric.

Vol. (45) No. (4) 2017

$$
\sigma_{A}^{2}=2 \sigma_{G C A}^{2} \cdot \sigma_{D}^{2}=\sigma_{S C A}^{2} \cdot \sigma_{E}^{2}=M s e / r
$$

و اختبرت معنوياتها عن الصفر حسب طريقة Kempthorne (1957). قدرت نسبة التوريث بالمعنيين الو اسع (

$$
\% h_{b . s}^{2}=\frac{\sigma_{G}^{2}}{\sigma_{P}^{2}} \times 100 \text { '\% } \% h_{n . s}^{2}=\frac{\sigma_{A}^{2}}{\sigma_{P}^{2}} \times 100 ، \bar{a}=\sqrt{\frac{2 \sigma_{D}^{2}}{\sigma_{A}^{2}}}
$$

كما بأني:

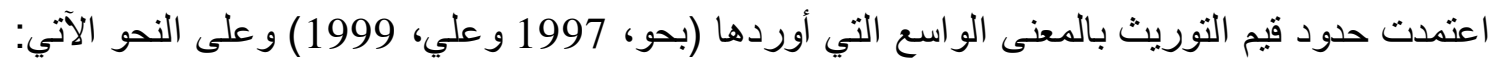
\% 40 > $\boldsymbol{h}_{\boldsymbol{b} . \boldsymbol{s}}^{\mathbf{2}}$

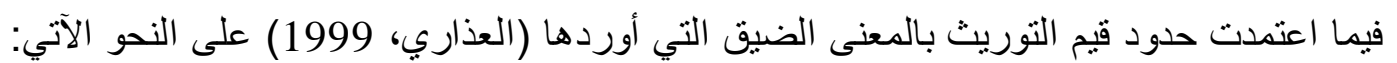
\% 20 > $\boldsymbol{h}_{\text {n.s }}^{2}$

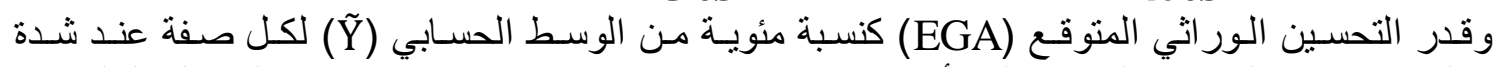
الانتخاب لـ 10\% من النباتات بالطريقة التي أوضحها (Kempthorne، من كنة 1969) عن طريق المعادلة التالية:EGA \% $=\left[\left(\mathrm{K} \mathrm{h}^{2} \sqrt{ } \sigma^{2} \mathrm{p}\right) / \tilde{\mathrm{Y}}\right] \times 100$.

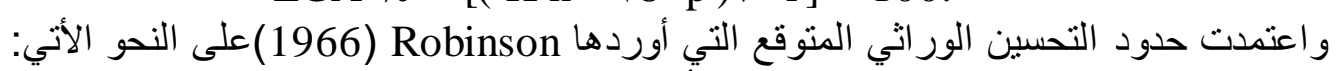

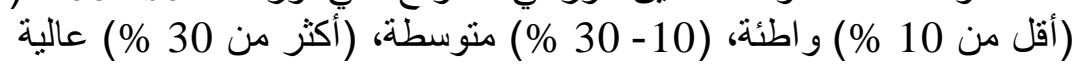

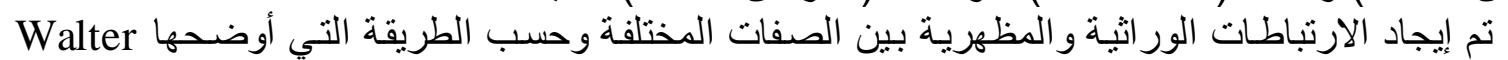

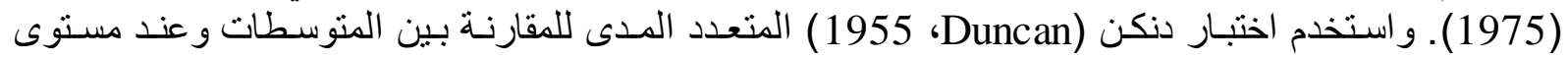

\section{النتائج و المناقشة}

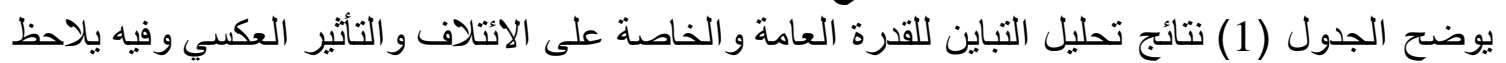

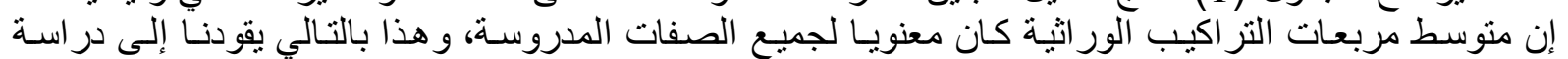

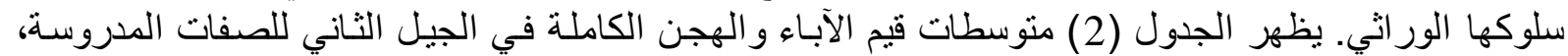

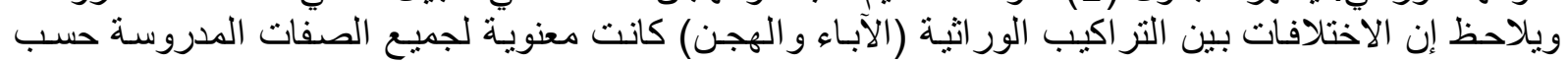

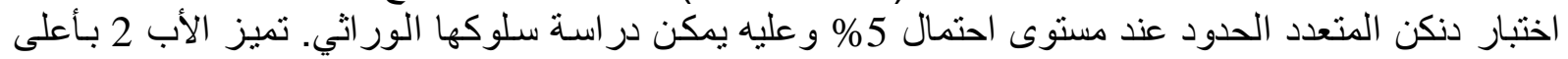

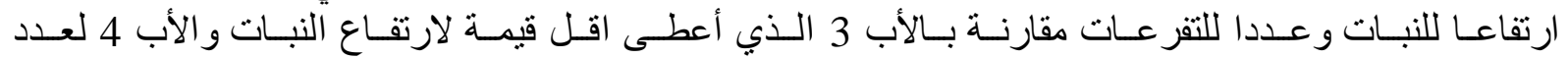

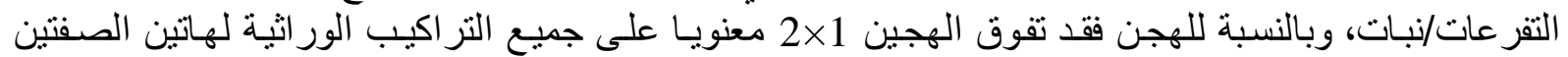

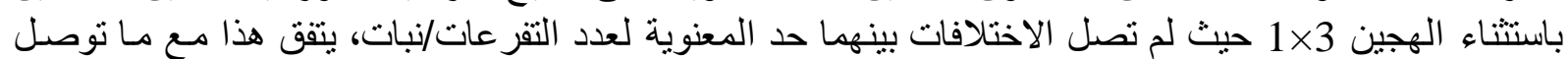

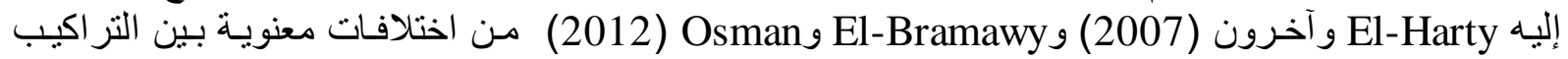
الور اثثية لارتفاع النبات و عدد الترون (التفرعات/نات التبات.

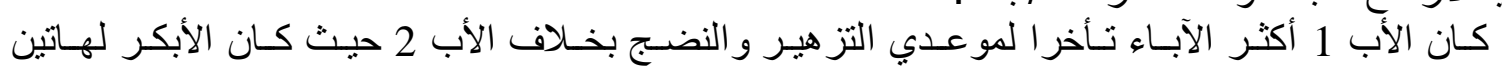

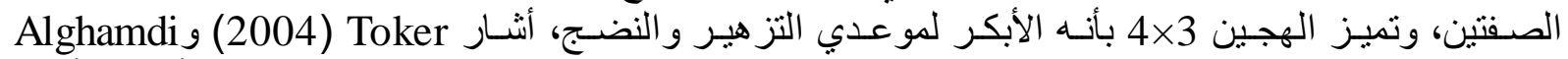

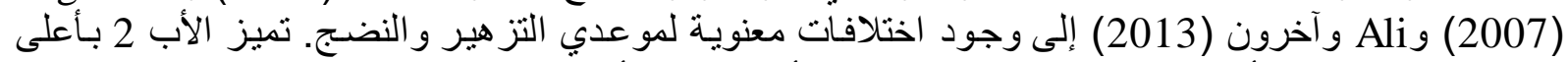

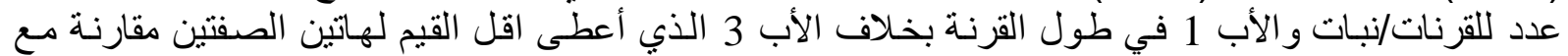

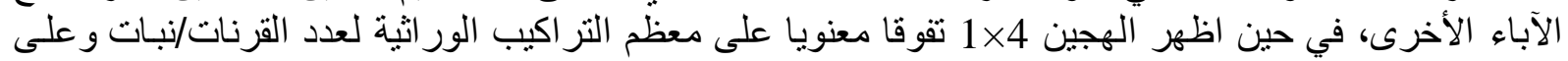

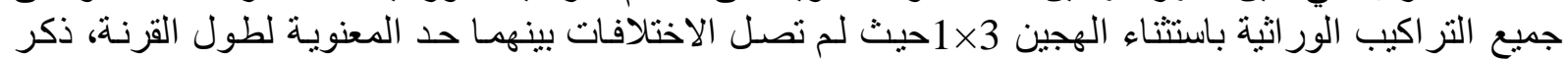

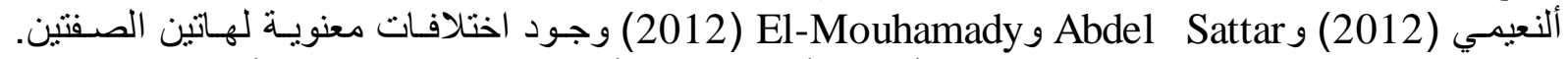

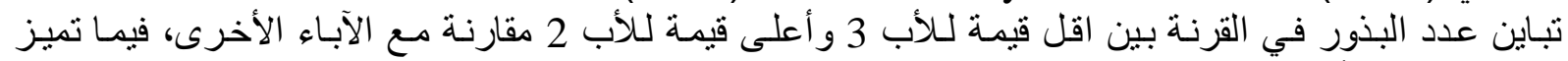

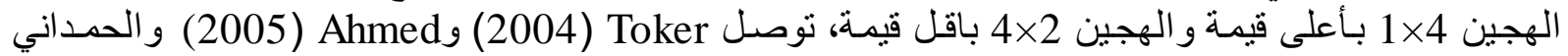

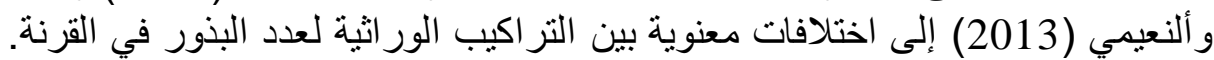

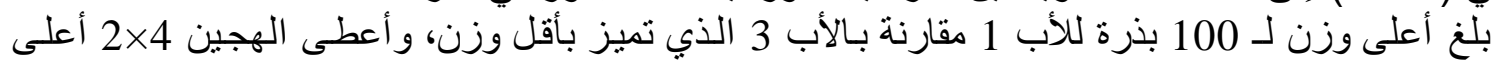

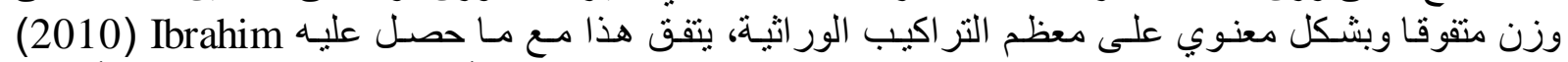

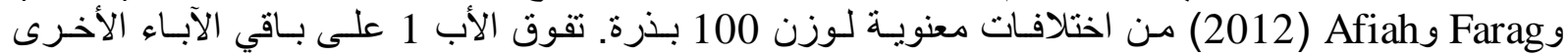

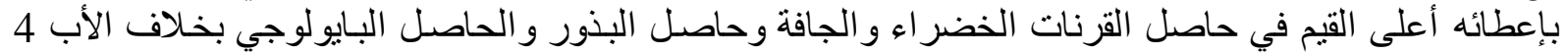

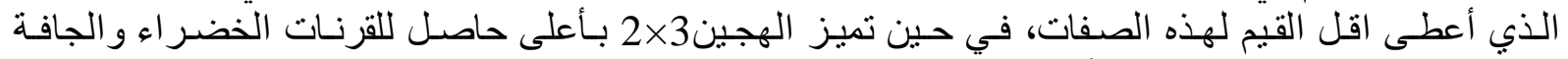

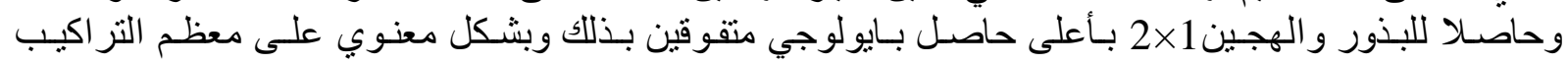


Mesopotamia J. of Agric.

Vol. (45) No. (4) 2017
ISSN: 2224 - 9796 (Online)

ISSN: 1815 - $316 \mathrm{X}$ (Print)
مجلـــة زراعـة الــر افديـن

المجلد (45) العدد (4) 2017

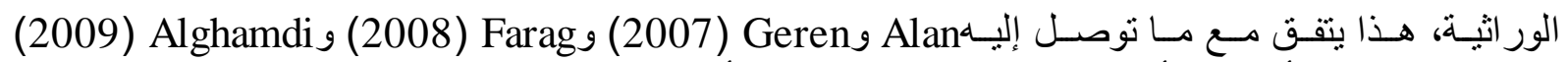

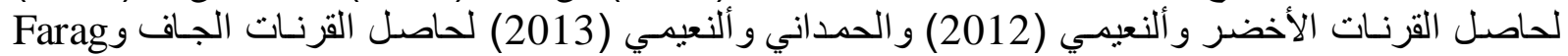

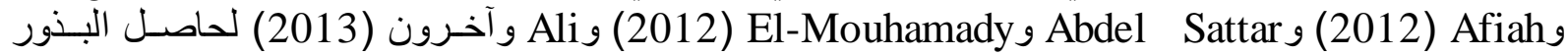
و الحمداني (2012 a,b) للحاصل البايولوجي. ييتِّن الجدول (3) قيم التدهور بسبب التربية الداخلية لكل هجين من هجن الجيل الثناني في الصفات

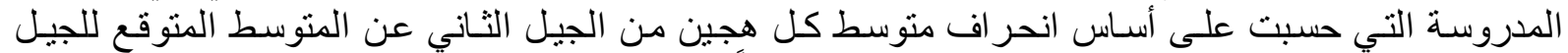

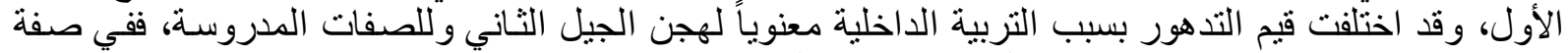

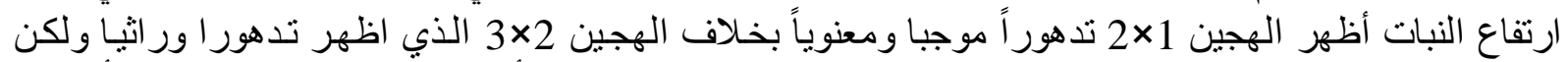

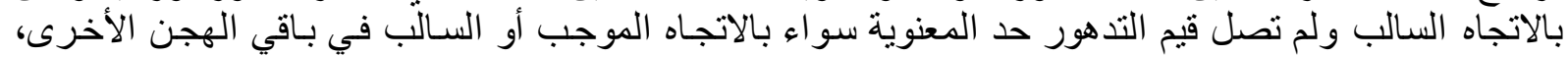

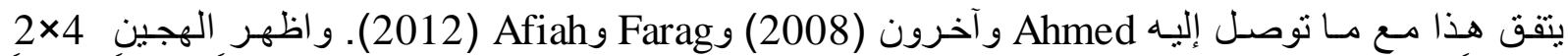

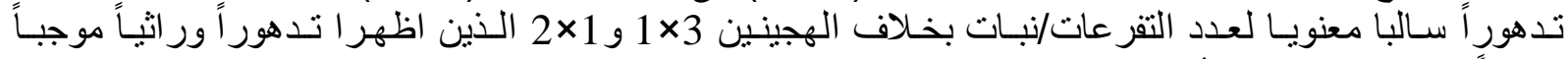

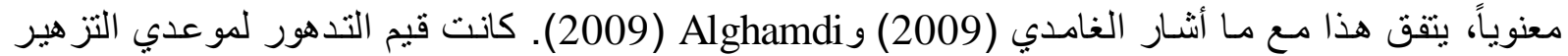

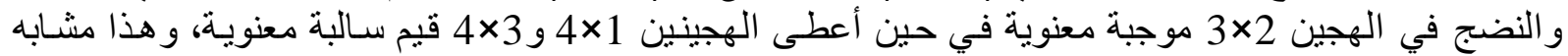

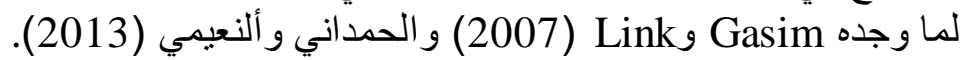

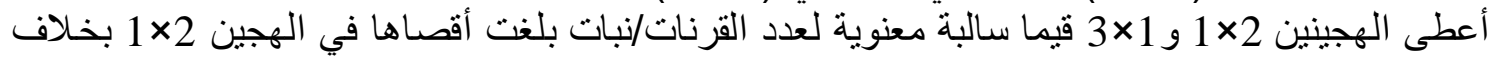

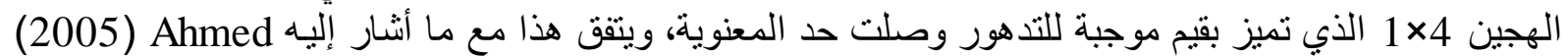

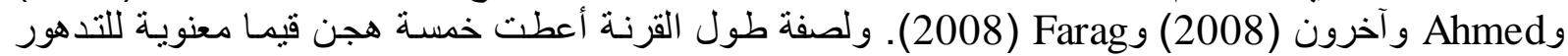

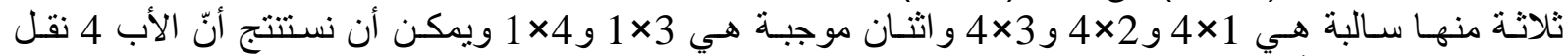

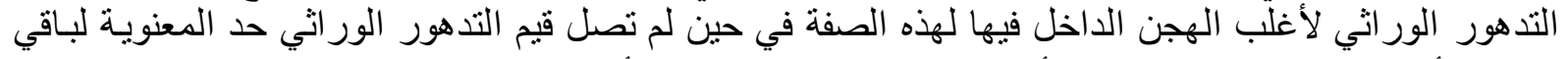

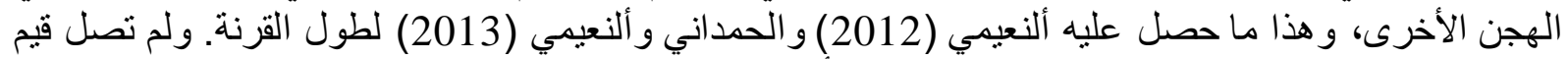

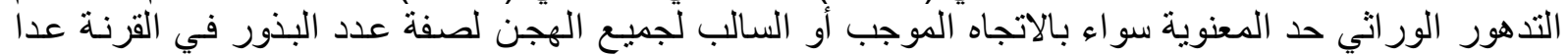

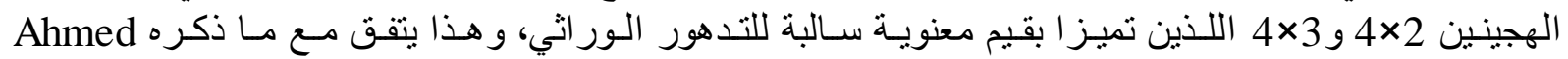

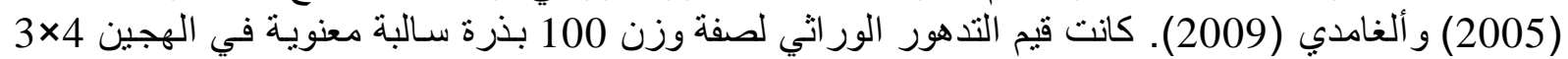

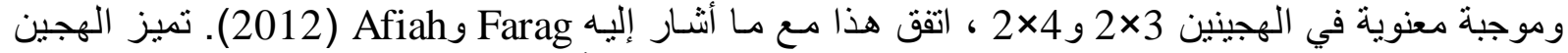

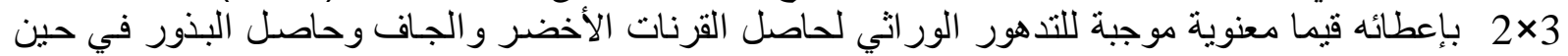

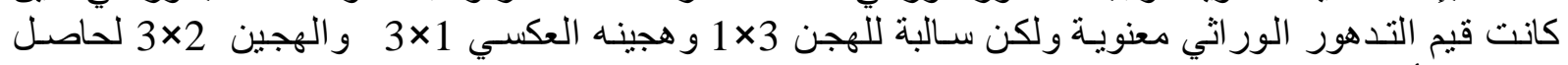

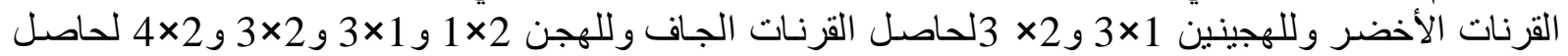

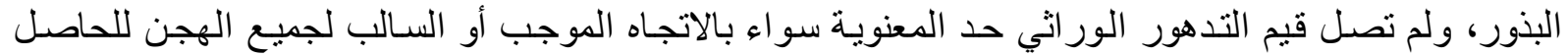
البايولوجي باستثناء الهجينين 1×3 و 2× 3 اللذين اظهر الثيم سالبة للتدهور الور اثي وصلت حد المعنوية لهذه

الصفة.

الجدول (1): تحليل تباين قدرة الائتلاف العامة والخاصة و التأثير العكسي ( الآباء + هجن الجيل الثاني) للصفات المدروسة.

Table(1): Analysis of variance of general, specific combining ability and reciprocal effect (parents $+\mathrm{F}_{2}$ hybrids) for studied characters.

\begin{tabular}{|c|c|c|c|c|c|c|c|}
\hline \multicolumn{6}{|c|}{ منوسط المربعات Mean Squares } & \multirow[b]{2}{*}{$\begin{array}{c}\text { الحرية } \\
\text { Degrees } \\
\text { of } \\
\text { Freedom } \\
\text { (d.f.) }\end{array}$} & \multirow[b]{2}{*}{$\begin{array}{c}\text { مصادر الاختلاف } \\
\text { Sources of Variation } \\
\text { (S.O.V.) }\end{array}$} \\
\hline $\begin{array}{c}\text { القرنة } \\
\text { القة) } \\
\text { Pod } \\
\text { Podth } \\
\text { length) }\end{array}$ & $\begin{array}{c}\text { القرنات } \\
\text { النبات / } \\
\text { No. of } \\
\text { pods } \\
\text { / Plant }\end{array}$ & $\begin{array}{c}\text { موعد } \\
\text { النضوم) } \\
\text { Date } \\
\text { maturity } \\
\text { (days) }\end{array}$ & $\begin{array}{c}\text { التز هير } \\
\text { (يوم) } \\
\text { Date } \\
\text { flowering } \\
\text { (days) }\end{array}$ & $\begin{array}{c}\text { التفر عات } \\
\text { / نبات / } \\
\text { No. of } \\
\text { branches } \\
\text { / Plant }\end{array}$ & $\begin{array}{c}\text { ارتفاع النبات) } \\
\text { Plant } \\
\text { Plant } \\
\text { height } \\
\text { (cm) }\end{array}$ & & \\
\hline 1.582 & 31.019 & 1.836 & 19.808 & 3.623 & 96.817 & 2 & $\begin{array}{c}\text { المكررات } \\
\text { Replications }\end{array}$ \\
\hline $\begin{array}{c}37.594 \\
* *\end{array}$ & $\begin{array}{c}100.865 \\
* *\end{array}$ & $\begin{array}{c}65.450 \\
* *\end{array}$ & $\begin{array}{c}72.709 \\
* *\end{array}$ & $\begin{array}{c}8.347 \\
* *\end{array}$ & $\begin{array}{c}332.387 \\
* *\end{array}$ & 15 & التر اكيب الور اثية \\
\hline $\begin{array}{c}27.765 \\
* *\end{array}$ & $\begin{array}{c}60.539 \\
* *\end{array}$ & $\begin{array}{c}11.670 \\
* *\end{array}$ & 9.763 & $\begin{array}{c}6.560 \\
* *\end{array}$ & $\begin{array}{c}219.904 \\
* *\end{array}$ & 3 & $\begin{array}{c}\text { قدرة الائتلاف العامة } \\
\text { General combining } \\
\text { ability }\end{array}$ \\
\hline
\end{tabular}


Mesopotamia J. of Agric.

Vol. (45) No. (4) 2017
ISSN: 2224 - 9796 (Online)

ISSN: 1815 - 316 X (Print)
مجلــة زراعــة الـر افديـن

المجلد (45) العدد (4) 2017

\begin{tabular}{|c|c|c|c|c|c|c|c|}
\hline $\begin{array}{l}4.404 \\
* *\end{array}$ & 23.239 & $\begin{array}{c}15.317 \\
* *\end{array}$ & $\begin{array}{c}12.199 \\
*\end{array}$ & 1.182 & $\begin{array}{l}58.052 \\
*\end{array}$ & 6 & $\begin{array}{c}\text { قدرة الائتلاف الخاصة } \\
\text { Specific combining } \\
\text { ability }\end{array}$ \\
\hline $\begin{array}{c}13.041 \\
* *\end{array}$ & $\begin{array}{c}30.545 \\
*\end{array}$ & $\begin{array}{c}33.389 \\
* *\end{array}$ & $\begin{array}{c}43.510 \\
* *\end{array}$ & $\begin{array}{c}2.493 \\
* *\end{array}$ & $\begin{array}{c}108.985 \\
* *\end{array}$ & 6 & $\begin{array}{c}\text { التأثير العكسي } \\
\text { Reciprocal effect }\end{array}$ \\
\hline 0.687 & 27.231 & 1.434 & 8.985 & 1.449 & 46.370 & 30 & $\begin{array}{c}\text { الخطا التجريبي } \\
\text { Error }\end{array}$ \\
\hline 1.649 & 0.908 & 0.188 & 0.183 & 2.174 & 1.199 & & مكونات تباين G.C.A.A.A.A. مكونات \\
\hline
\end{tabular}

\begin{tabular}{|c|c|c|c|c|c|c|c|}
\hline \multicolumn{6}{|c|}{ متوسط المربعات Mean Squares } & \multirow[b]{2}{*}{$\begin{array}{c}\text { درجاتة } \\
\text { الحرية } \\
\text { Degrees } \\
\text { of } \\
\text { Freedom } \\
\text { (d.f.) }\end{array}$} & \multirow[b]{2}{*}{$\begin{array}{c}\text { Sصادر الاختلاف } \\
\text { Sources of } \\
\text { Variation } \\
\text { (S.O.V.) }\end{array}$} \\
\hline $\begin{array}{c}\text { البايولوجي } \\
\text { (غاصنات) } \\
\text { Biological } \\
\text { yield } \\
\text { (gm/plant) }\end{array}$ & $\begin{array}{c}\text { حاصل البذور) } \\
\text { (غم/نبات } \\
\text { Seeds } \\
\text { yield } \\
\text { (gm/plant) }\end{array}$ & $\begin{array}{c}\text { القرنات الجافل } \\
\text { الجاتنات) } \\
\text { Dry pods } \\
\text { yield } \\
\text { (gm/plant) }\end{array}$ & $\begin{array}{c}\text { القرناتل } \\
\text { الأخضر: } \\
\text { الخاتر) } \\
\text { Green } \\
\text { pods yield } \\
\text { (gm/plant) }\end{array}$ & $\begin{array}{c}\text { وزنرة } 100 \\
\text { (غذ) } \\
100 \text { seed } \\
\text { weight } \\
(\mathrm{gm})\end{array}$ & $\begin{array}{c}\text { البنور } \\
\text { القدنة } \\
\text { فرد } \\
\text { No. of } \\
\text { seeds } \\
\text { per } \\
\text { pod }\end{array}$ & & \\
\hline 116424.300 & 327.765 & 609.594 & 18249.656 & 29.483 & 1.457 & 2 & $\begin{array}{c}\text { المكررات } \\
\text { Replications }\end{array}$ \\
\hline $\begin{array}{c}158475.600 \\
* * \\
\end{array}$ & $\begin{array}{c}1633.641 \\
* * \\
\end{array}$ & $\begin{array}{c}2623.447 \\
* * \\
\end{array}$ & $\begin{array}{c}47159.880 \\
* * \\
\end{array}$ & $\begin{array}{c}1179.797 \\
* * \\
\end{array}$ & $\begin{array}{c}3.614 \\
* \\
\end{array}$ & 15 & التراكيب الور اثية \\
\hline $\begin{array}{c}96881.830 \\
* *\end{array}$ & $\begin{array}{c}1286.185 \\
* *\end{array}$ & $\begin{array}{l}2082.459 \\
* *\end{array}$ & $\begin{array}{l}36864.905 \\
* *\end{array}$ & $\begin{array}{c}1032.187 \\
* *\end{array}$ & 1.477 & 3 & $\begin{array}{c}\text { قدرة الائتلاف العامة } \\
\text { General combining } \\
\text { ability }\end{array}$ \\
\hline $\begin{array}{l}36051.390 \\
*\end{array}$ & $\begin{array}{c}210.514 \\
* *\end{array}$ & $\begin{array}{c}400.040 \\
*\end{array}$ & $\begin{array}{c}9443.318 \\
*\end{array}$ & 92.350 & 0.809 & 6 & $\begin{array}{l}\text { قدرة الائتلاف الخاصنة } \\
\text { Specific } \\
\text { combining ability }\end{array}$ \\
\hline $\begin{array}{c}47570.730 \\
*\end{array}$ & $\begin{array}{c}507.760 \\
* *\end{array}$ & $\begin{array}{c}744.935 \\
* *\end{array}$ & $\begin{array}{c}11424.130 \\
* *\end{array}$ & $\begin{array}{c}374.720 \\
* *\end{array}$ & $\begin{array}{c}1.463 \\
*\end{array}$ & 6 & $\begin{array}{c}\text { التأثير العكسي } \\
\text { Reciprocal effect }\end{array}$ \\
\hline 38394.090 & 145.243 & 375.345 & 6596.932 & 185.578 & 1.423 & 30 & الخطاً التجريبي \\
\hline 0.903 & 1.908 & 1.779 & 1.196 & 7.956 & 0.748 & & G.C.A. مكونات تباين S.C.A.A.A مكوناتين \\
\hline
\end{tabular}

*,** Significant at $\mathrm{P}$

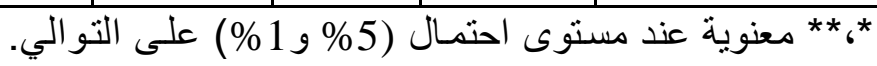

(5\% and $1 \%$ ) respectively.

الجدول (2): قيم منوسطات الآباء و الهجن الكاملة في الجيل الثانيF2 للصفات المدروسة.

Table(2): Means values of parents and complete in $\mathrm{F}_{2}$ hybrids for studied characters.

\begin{tabular}{|c|c|c|c|c|c|c|}
\hline $\begin{array}{c}\text { القرنة } \\
\text { القر) } \\
\text { Pod } \\
\text { Pongth } \\
\text { (cm) }\end{array}$ & $\begin{array}{c}\text { القرنات } \\
\text { عدات / } \\
\text { No. of } \\
\text { pods / } \\
\text { Plant } \\
\end{array}$ & $\begin{array}{c}\text { موعد } \\
\text { النضج) } \\
\text { (يوم) } \\
\text { Date } \\
\text { maturity } \\
\text { (days) }\end{array}$ & $\begin{array}{c}\text { موعزير (يوم) } \\
\text { (يلزير } \\
\text { Date } \\
\text { flowering } \\
\text { (days) }\end{array}$ & $\begin{array}{c}\text { التقر عات / } 1 \text { عبات } \\
\text { No. of } \\
\text { branches / } \\
\text { Plant }\end{array}$ & $\begin{array}{c}\text { النبات } \\
\text { النفاع) } \\
\text { Plant } \\
\text { height } \\
\text { (cm) }\end{array}$ & التر اكيب الور اثية \\
\hline $19.483 \mathrm{~b}$ & 18.726 b-d & $168.226 \mathrm{~b}$ & $97.733 \mathrm{bc}$ & $5.996 \mathrm{~cd}$ & $73.956 \mathrm{~b}-\mathrm{d}$ & $\begin{array}{c}\text { 1- French } \\
\text { (Aguadulce) }\end{array}$ \\
\hline $15.890 \mathrm{~d}$ & $28.546 \mathrm{a}$ & $157.860 \mathrm{gh}$ & $87.960 \mathrm{~d}-\mathrm{f}$ & $6.590 \mathrm{bc}$ & $80.940 \mathrm{bc}$ & 2- Syrian (Shami) \\
\hline 13.686 ef & $14.043 \mathrm{c}-\mathrm{e}$ & $162.436 \mathrm{~d}$ & $92.653 \mathrm{c}-\mathrm{e}$ & $5.533 \mathrm{c}-\mathrm{e}$ & $63.893 \mathrm{de}$ & 3- Spain \\
\hline $14.833 \mathrm{de}$ & $15.213 \mathrm{c}-\mathrm{e}$ & $165.120 \mathrm{c}$ & $95.563 \mathrm{bc}$ & 3.523 ef & 70.190 b-e & 4-Holland \\
\hline $18.013 \mathrm{c}$ & $12.046 \mathrm{c}-\mathrm{e}$ & $166.213 \mathrm{bc}$ & $96.326 \mathrm{bc}$ & $5.916 \mathrm{~cd}$ & $82.603 \mathrm{~b}$ & $1 \mathrm{X} 2$ \\
\hline $20.996 \mathrm{a}$ & $11.373 \mathrm{c}-\mathrm{e}$ & $162.130 \mathrm{de}$ & $93.313 b-d$ & $8.280 \mathrm{ab}$ & $68.886 \mathrm{c}-\mathrm{e}$ & $1 \mathrm{X} 3$ \\
\hline
\end{tabular}


Mesopotamia J. of Agric.

Vol. (45) No. (4) 2017
ISSN: 2224 - 9796 (Online)

ISSN: 1815 - $316 \mathrm{X}$ (Print)

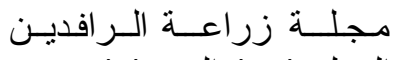

المجلد (45) العدد (4) 2017

\begin{tabular}{|c|c|c|c|c|c|c|}
\hline $21.060 \mathrm{a}$ & $25.536 \mathrm{ab}$ & $161.536 \mathrm{de}$ & $92.980 \mathrm{c}-\mathrm{e}$ & $6.803 \mathrm{bc}$ & $72.926 \mathrm{~b}-\mathrm{d}$ & $1 \mathrm{X} 4$ \\
\hline $16.166 \mathrm{~d}$ & $21.406 \mathrm{a}-\mathrm{c}$ & $158.093 \mathrm{gh}$ & $91.976 \mathrm{c}-\mathrm{e}$ & $9.136 \mathrm{a}$ & $94.323 \mathrm{a}$ & $2 \mathrm{X} 1$ \\
\hline $16.056 \mathrm{~d}$ & $17.483 \mathrm{~b}-\mathrm{d}$ & $160.020 \mathrm{e}-\mathrm{g}$ & $87.626 \mathrm{~d}-\mathrm{f}$ & $6.083 \mathrm{~b}-\mathrm{d}$ & $79.873 \mathrm{bc}$ & $2 \mathrm{X} 3$ \\
\hline $15.673 \mathrm{~d}$ & $16.903 \mathrm{~b}-\mathrm{d}$ & $158.306 \mathrm{f}-\mathrm{h}$ & $88.163 \mathrm{~d}-\mathrm{f}$ & $2.230 \mathrm{f}$ & $66.210 \mathrm{de}$ & $2 \mathrm{X} 4$ \\
\hline $15.140 \mathrm{~d}$ & $5.916 \mathrm{e}$ & $167.320 \mathrm{~b}$ & $97.330 \mathrm{bc}$ & $6.323 \mathrm{~b}-\mathrm{d}$ & $59.523 \mathrm{ef}$ & $3 \mathrm{X} 1$ \\
\hline $13.223 \mathrm{f}$ & $21.450 \mathrm{a}-\mathrm{c}$ & $174.246 \mathrm{a}$ & $103.366 \mathrm{a}$ & $4.956 \mathrm{c}-\mathrm{e}$ & $49.186 \mathrm{f}$ & $3 \mathrm{X} 2$ \\
\hline $11.226 \mathrm{gh}$ & $13.736 \mathrm{c}-\mathrm{e}$ & $166.556 \mathrm{bc}$ & $98.996 \mathrm{ab}$ & $4.096 \mathrm{~d}-\mathrm{f}$ & $61.203 \mathrm{de}$ & $3 \mathrm{X} 4$ \\
\hline $12.463 \mathrm{fg}$ & $10.290 \mathrm{de}$ & $160.323 \mathrm{~d}-\mathrm{f}$ & $87.426 \mathrm{ef}$ & $5.743 \mathrm{c}-\mathrm{e}$ & $72.903 \mathrm{~b}-\mathrm{d}$ & $4 \mathrm{X} 1$ \\
\hline $9.880 \mathrm{hi}$ & $16.086 \mathrm{~b}-\mathrm{d}$ & $162.123 \mathrm{de}$ & $94.206 \mathrm{bc}$ & $5.796 \mathrm{c}-\mathrm{e}$ & $73.913 \mathrm{~b}-\mathrm{d}$ & $4 \mathrm{X} 2$ \\
\hline $9.413 \mathrm{i}$ & $13.190 \mathrm{c}-\mathrm{e}$ & $157.103 \mathrm{~h}$ & $85.883 \mathrm{f}$ & $4.876 \mathrm{c}-\mathrm{e}$ & $70.246 \mathrm{~b}-\mathrm{e}$ & $4 \mathrm{X} 3$ \\
\hline
\end{tabular}

\begin{tabular}{|c|c|c|c|c|c|c|}
\hline $\begin{array}{c}\text { البايولوجي } \\
\text { الحاصلنات) } \\
\text { Biological } \\
\text { yield } \\
\text { (gm/plant) }\end{array}$ & $\begin{array}{c}\text { البذور (غاصلنات) } \\
\text { Seeds } \\
\text { yield } \\
\text { (gm/plant) } \\
\end{array}$ & $\begin{array}{c}\text { حاصل القرنات } \\
\text { الجات (غبات) } \\
\text { Dry pods } \\
\text { yield } \\
\text { (gm/plant) }\end{array}$ & 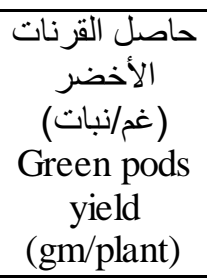 & $\begin{array}{c}\text { بذرة (غم) } 100 \\
100 \text { seed } \\
\text { weight } \\
\text { (gm) }\end{array}$ & $\begin{array}{c}\text { عدد البذور } \\
\text { القرنة } \\
\text { No. of } \\
\text { seeds per } \\
\text { pod }\end{array}$ & التر اكيب الور اثية \\
\hline $997.340 \mathrm{a}$ & $98.050 \mathrm{a}$ & $126.623 \mathrm{a}$ & $571.313 \mathrm{a}$ & $163.736 \mathrm{a}$ & $5.030 \mathrm{ab}$ & $\begin{array}{l}\text { 1- French } \\
\text { (Aguadulce) }\end{array}$ \\
\hline $879.526 \mathrm{a}-\mathrm{c}$ & $84.406 \mathrm{ab}$ & $107.420 \mathrm{a}-\mathrm{c}$ & 439.846 a-d & 134.036 b-e & $5.533 \mathrm{ab}$ & 2- Syrian (Shami) \\
\hline $741.763 \mathrm{a}-\mathrm{d}$ & $37.496 \mathrm{fg}$ & $57.253 \mathrm{~d}-\mathrm{f}$ & $326.523 \mathrm{~d}-\mathrm{f}$ & $98.670 \mathrm{fg}$ & $4.146 \mathrm{a}-\mathrm{d}$ & 3- Spain \\
\hline $438.440 \mathrm{~d}$ & $35.460 \mathrm{fg}$ & $44.496 \mathrm{ef}$ & $203.656 \mathrm{f}$ & $129.413 \mathrm{c}-\mathrm{e}$ & $4.703 \mathrm{ab}$ & 4-Holland \\
\hline 758.416 a-d & 64.570 b-e & $88.336 \mathrm{~cd}$ & 414.456 b-e & 146.050 a-c & $4.673 \mathrm{ab}$ & $1 \mathrm{X} 2$ \\
\hline 644.590 a-d & $46.453 \mathrm{~d}-\mathrm{g}$ & $58.763 \mathrm{~d}-\mathrm{f}$ & 294.416 d-f & 129.523 c-e & $5.593 \mathrm{ab}$ & $1 \mathrm{X} 3$ \\
\hline $950.950 \mathrm{ab}$ & $64.250 \mathrm{~b}-\mathrm{e}$ & 89.646 b-d & $394.560 \mathrm{c}-\mathrm{e}$ & $126.416 \mathrm{c}-\mathrm{e}$ & $6.046 \mathrm{a}$ & $1 \mathrm{X} 4$ \\
\hline $986.243 \mathrm{a}$ & $71.416 \mathrm{bc}$ & 99.770 a-c & $479.893 \mathrm{a}-\mathrm{c}$ & 142.756 a-c & $5.313 \mathrm{ab}$ & $2 \mathrm{X} 1$ \\
\hline $936.053 \mathrm{ab}$ & $93.940 \mathrm{a}$ & $124.123 \mathrm{ab}$ & $547.896 \mathrm{ab}$ & $141.430 \mathrm{a}-\mathrm{d}$ & $4.690 \mathrm{ab}$ & $2 \times 3$ \\
\hline $458.953 \mathrm{~d}$ & 67.893 b-d & 80.273 c-e & 276.890 ef & $158.203 \mathrm{ab}$ & $5.360 \mathrm{ab}$ & $2 \mathrm{X} 4$ \\
\hline $375.146 \mathrm{~d}$ & $34.440 \mathrm{fg}$ & $42.823 \mathrm{f}$ & $185.306 \mathrm{f}$ & $116.043 \mathrm{~d}-\mathrm{f}$ & $4.213 \mathrm{a}-\mathrm{d}$ & $3 \mathrm{X} 1$ \\
\hline $387.203 \mathrm{~d}$ & $28.446 \mathrm{~g}$ & $39.526 \mathrm{f}$ & $217.190 \mathrm{f}$ & 111.756 ef & $4.763 \mathrm{ab}$ & $3 \times 2$ \\
\hline $460.583 \mathrm{~d}$ & $25.780 \mathrm{~g}$ & $40.793 \mathrm{f}$ & $201.870 \mathrm{f}$ & $87.623 \mathrm{~g}$ & $3.636 \mathrm{~b}-\mathrm{d}$ & $3 \mathrm{X} 4$ \\
\hline 581.136 b-d & $55.183 \mathrm{c}-\mathrm{f}$ & $75.106 \mathrm{c}-\mathrm{f}$ & $317.496 \mathrm{~d}-\mathrm{f}$ & 135.436 b-e & $4.440 \mathrm{a}-\mathrm{c}$ & $4 \mathrm{X} 1$ \\
\hline $450.786 \mathrm{~d}$ & $33.430 \mathrm{fg}$ & 49.470 ef & $231.556 \mathrm{f}$ & $124.783 \mathrm{c}-\mathrm{e}$ & $2.036 \mathrm{~d}$ & $4 X 2$ \\
\hline $551.573 \mathrm{~cd}$ & $44.290 \mathrm{e}-\mathrm{g}$ & $56.206 \mathrm{~d}-\mathrm{f}$ & 261.383 ef & 134.796 b-e & $2.366 \mathrm{~cd}$ & $4 X 3$ \\
\hline
\end{tabular}

* القيم المتبو عة بالحرف الأبجدي نفسه لكل صفة لا نختلف عن بعضها معنويا حسب اختبار دنكن المتعدد الحدود

$$
\text { و عند مستوى احتمال }
$$

* Means followed by the same letter within a column do not differ significantly from each other using Duncan's multiple range test at 5\% level.

$$
\text { الجدول (3): قيم التدهور نتيجة التربية الداخلية لهن الجيل الثاني F في الصفات المدروسة. }
$$

\begin{tabular}{|c|c|c|c|c|c|c|}
\hline $\begin{array}{c}\text { طونة } \\
\text { القزنة } \\
\text { Pod } \\
\text { Pod } \\
\text { length } \\
\text { (cm) }\end{array}$ & $\begin{array}{c}\text { القرنات } \\
\text { عدات / } \\
\text { No. of } \\
\text { pods / } \\
\text { Plant }\end{array}$ & $\begin{array}{c}\text { موعد } \\
\text { النضج) } \\
\text { Date } \\
\text { Date } \\
\text { maturity } \\
\text { (days) }\end{array}$ & $\begin{array}{c}\text { التز هير } \\
\text { (يوم) } \\
\text { Date } \\
\text { flowering } \\
\text { (days) }\end{array}$ & $\begin{array}{c}\text { / التفر عات / } 1 \text { / } \\
\text { / } \\
\text { No. of } \\
\text { branches / } \\
\text { Plant }\end{array}$ & $\begin{array}{c}\text { النفات } \\
\text { النفاع) } \\
\text { Plant } \\
\text { height } \\
\text { (cm) }\end{array}$ & $\begin{array}{c}\text { الهجن } \\
\text { Hybrids }\end{array}$ \\
\hline 0.326 & $-11.590 *$ & 3.170 & 3.480 & -0.376 & 5.155 & $1 \mathrm{X} 2$ \\
\hline $4.411 *$ & -5.0117 & -3.201 & -1.880 & $2.515 *$ & -0.038 & $1 \mathrm{X} 3$ \\
\hline $4.475 *$ & $9.151 *$ & -3.795 & -2.213 & 1.038 & 4.001 & $1 \mathrm{X} 4$ \\
\hline-1.520 & -2.230 & -4.950 & -0.870 & $2.843 *$ & $16.875 *$ & $2 \mathrm{X} 1$ \\
\hline 1.268 & -3.811 & -0.1283 & -2.680 & 0.021 & 7.456 & $2 \times 3$ \\
\hline
\end{tabular}

Table(3): Inbreeding de pression values in $\mathrm{F}_{2}$ hybrids for studied characters. 
Mesopotamia J. of Agric.

Vol. (45) No. (4) 2017
ISSN: $2224-9796$ (Online)

ISSN: 1815 - 316 X (Print)

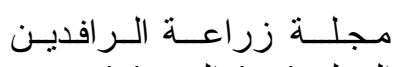

المجلد (45) العدد (4) 2017

\begin{tabular}{|c|c|c|c|c|c|c|}
\hline 0.311 & -4.976 & -3.183 & -3.598 & $-2.826 *$ & -9.355 & $2 X 4$ \\
\hline-1.445 & $-10.468 *$ & 1.988 & 2.136 & 0.558 & -9.401 & $3 \mathrm{X} 1$ \\
\hline-1.565 & 0.155 & $14.098^{* *}$ & $13.060 * *$ & -1.105 & $-23.230 * *$ & $3 \mathrm{X} 2$ \\
\hline-3.033 & -0.891 & 2.778 & 4.888 & -0.431 & -5.838 & $3 \mathrm{X} 4$ \\
\hline$-4.695 *$ & -6.680 & $-6.350 *$ & $-9.221 *$ & 0.983 & 0.830 & $4 X 1$ \\
\hline$-5.481 *$ & -5.793 & 0.633 & 2.445 & 0.740 & -1.651 & $4 X 2$ \\
\hline$-4.846^{*}$ & -1.438 & $-6.675 *$ & $-8.225 *$ & 0.348 & 3.205 & $4 X 3$ \\
\hline
\end{tabular}

\begin{tabular}{|c|c|c|c|c|c|c|}
\hline 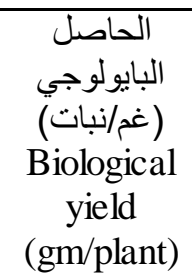 & $\begin{array}{c}\text { (غلبندر) } \\
\text { (غلنات) } \\
\text { Seeds } \\
\text { yield } \\
\text { (gm/plant) }\end{array}$ & 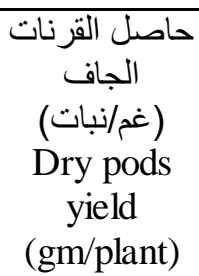 & $\begin{array}{c}\text { حاصل الخضرنات } \\
\text { الغر/نبات) } \\
\text { Green pods } \\
\text { yield } \\
\text { (gm/plant) }\end{array}$ & $\begin{array}{c}\text { بذرة (غر) } 100 \\
100 \text { seed } \\
\text { weight } \\
\text { (gm) }\end{array}$ & $\begin{array}{c}\text { عدد البذور في } \\
\text { No. of seeds } \\
\text { per pod }\end{array}$ & $\begin{array}{c}\text { الهجن } \\
\text { Hybrids }\end{array}$ \\
\hline-180.017 & $-26.658 *$ & -28.685 & -91.123 & -2.836 & -0.608 & $1 \mathrm{X} 2$ \\
\hline-224.962 & -21.320 & -33.175 & $-154.502 *$ & -1.680 & 1.005 & $1 \mathrm{X} 3$ \\
\hline 81.398 & -3.523 & -2.291 & -54.358 & -4.786 & 1.458 & $1 \mathrm{X} 4$ \\
\hline 47.810 & -19.811 & -17.251 & -25.686 & -6.130 & 0.031 & $2 \mathrm{X} 1$ \\
\hline 125.408 & $32.988 *$ & $41.786 *$ & $164.711 *$ & $25.076 *$ & -0.150 & $2 \times 3$ \\
\hline-200.030 & 7.960 & 4.315 & -44.861 & $26.478 *$ & 0.241 & $2 \mathrm{X} 4$ \\
\hline$-494.405 * *$ & $-33.333 *$ & $-49.115 * *$ & $-263.612 * *$ & -15.160 & -0.375 & $3 \mathrm{X} 1$ \\
\hline$-423.442 *$ & $-32.505 *$ & $-42.810 *$ & $-165.995 *$ & -4.596 & -0.076 & $3 \mathrm{X} 2$ \\
\hline-129.518 & -10.698 & -10.081 & -63.220 & $-26.418 *$ & -0.788 & $3 \mathrm{X} 4$ \\
\hline-136.753 & -11.571 & -10.453 & -69.988 & -11.138 & -0.426 & $4 \mathrm{X} 1$ \\
\hline-208.197 & $-26.503 *$ & -26.488 & -90.195 & -6.941 & $-3.081 * *$ & $4 \mathrm{X} 2$ \\
\hline-38.528 & 7.811 & 5.331 & -3.706 & 20.755 & $-2.058 *$ & $4 \mathrm{X} 3$ \\
\hline
\end{tabular}

*,** Significant at $\mathrm{P}(5 \%$ and $1 \%)$ respectively.

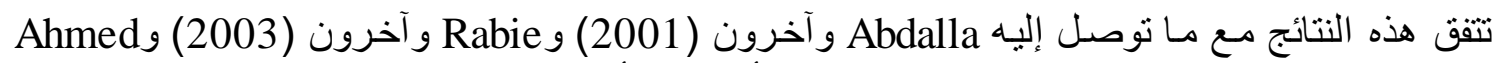

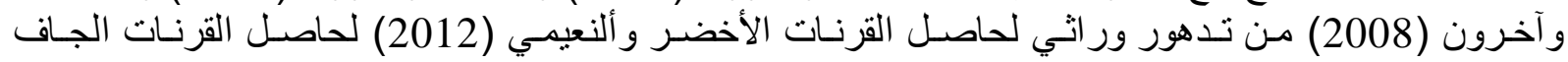

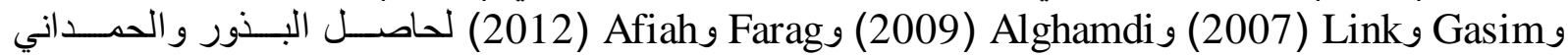

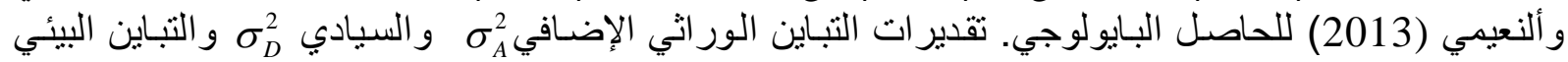

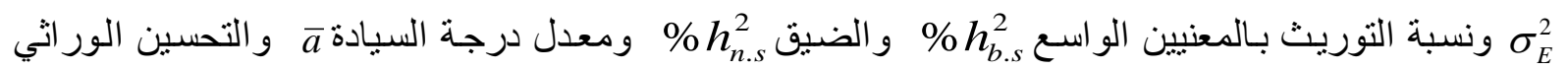

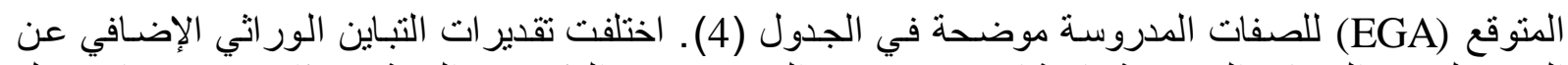

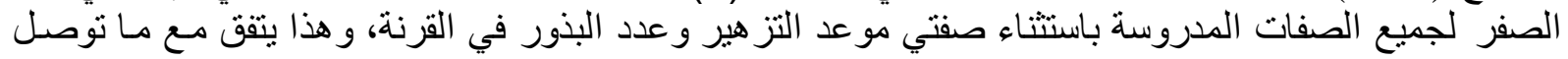

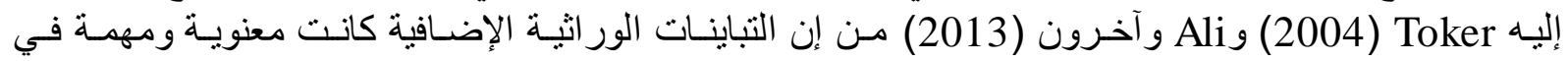

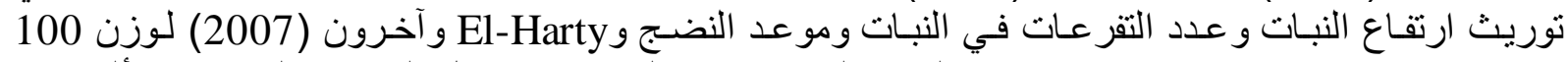

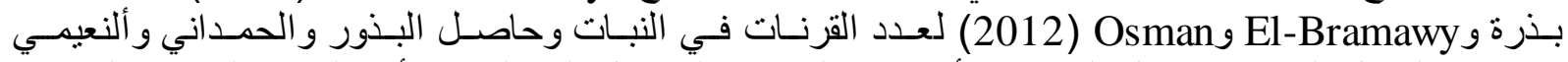

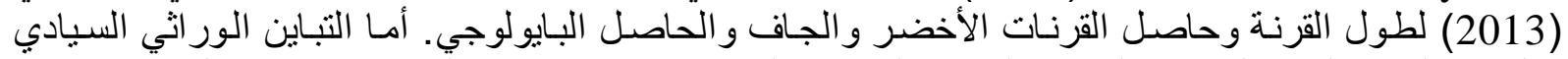

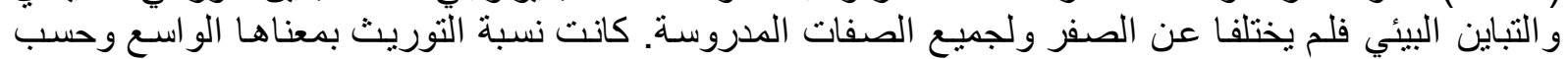

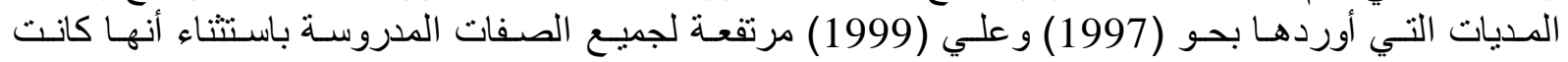

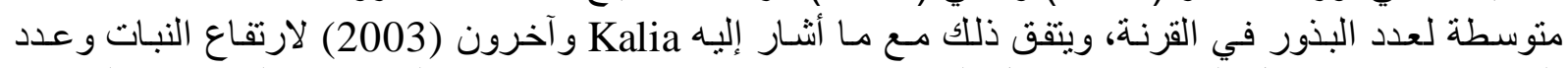

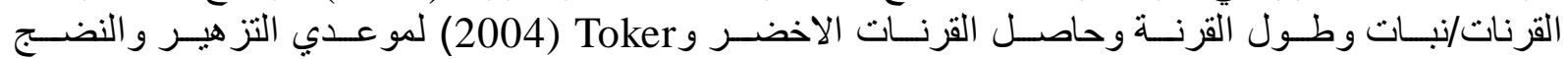

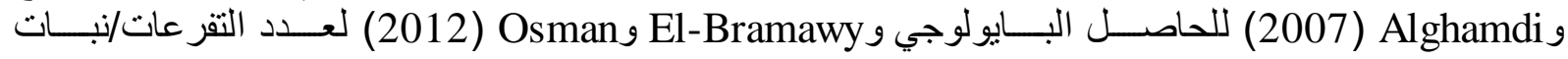
Abdel

Sattar 9

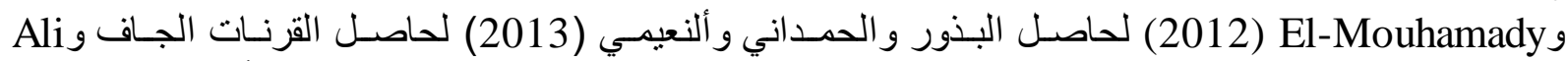

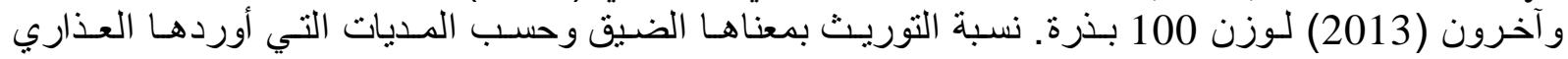

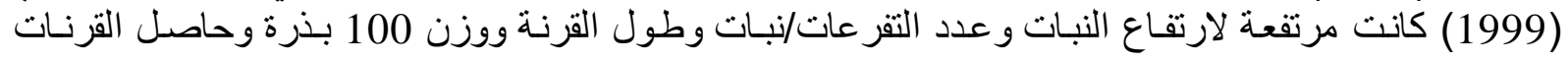


Mesopotamia J. of Agric.

Vol. (45) No. (4) 2017
ISSN: $2224-9796$ (Online)

ISSN: 1815 - $316 \mathrm{X}$ (Print)

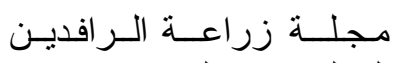

المجلد (45) العدد (4) 2017

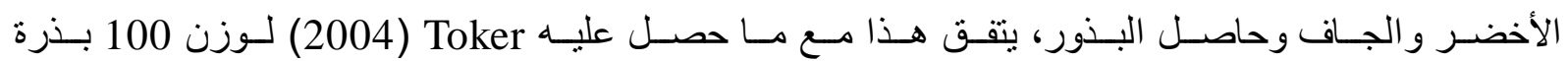

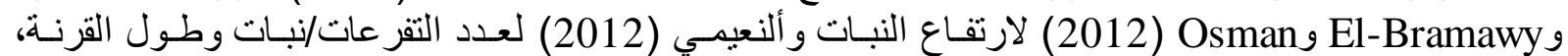

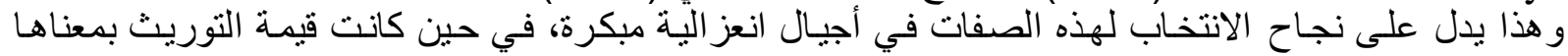

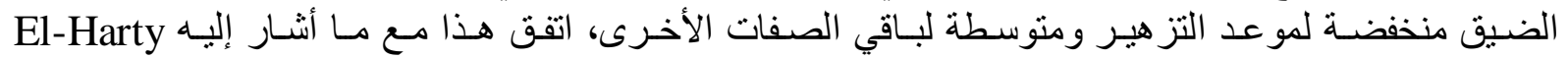

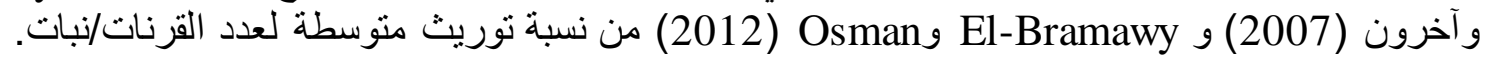

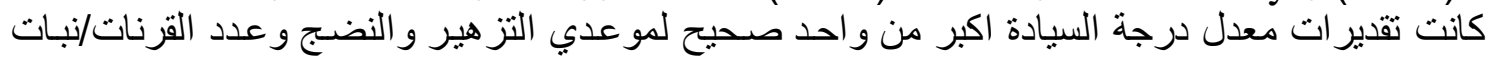

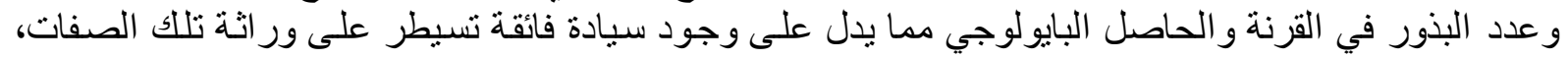

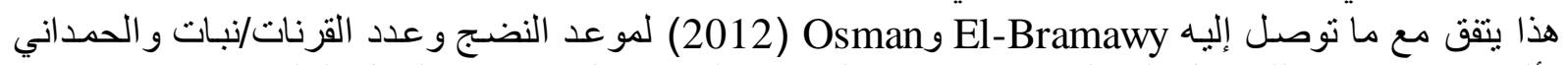

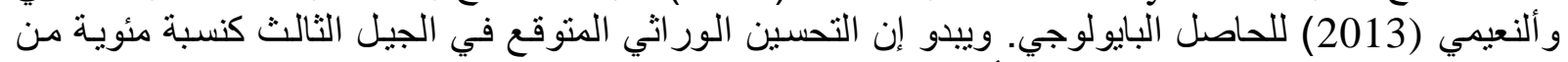

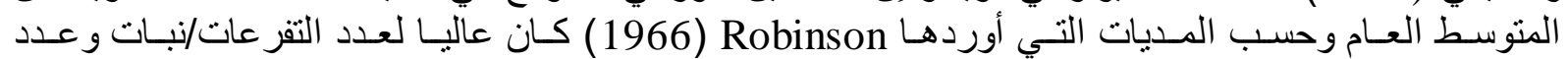

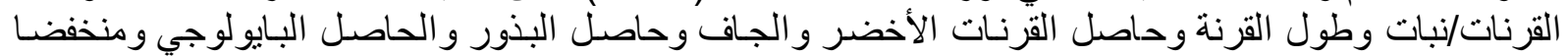

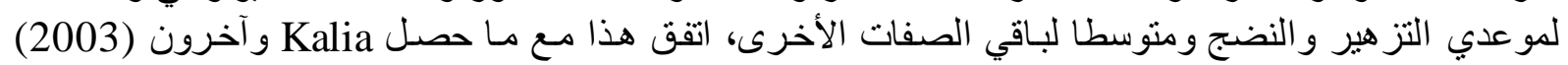

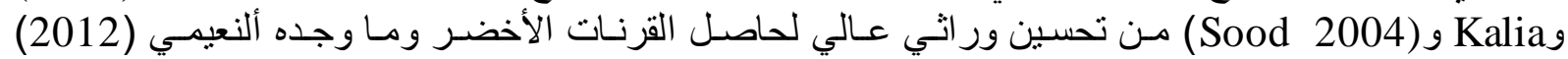

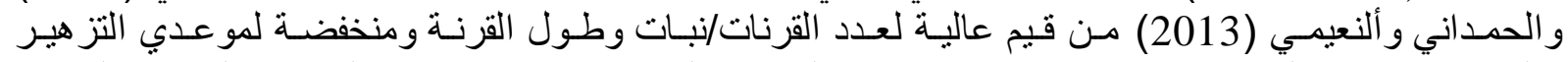

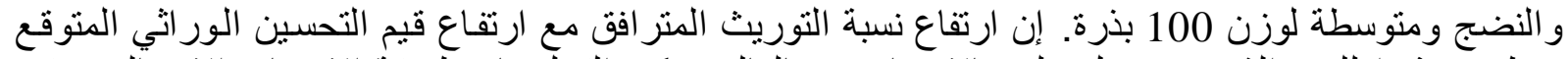

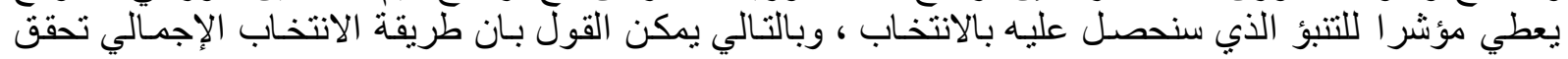

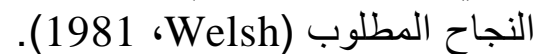
يتضح من الجدول (5) وجود ارتباط مظهري وور اثي موجب معنوي بين ارتفاع النبات وكل من صن صفات

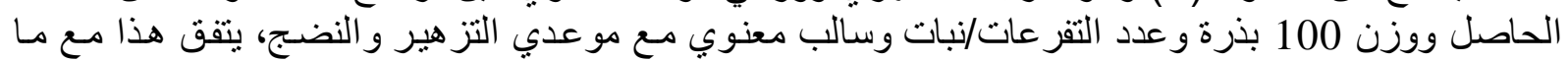

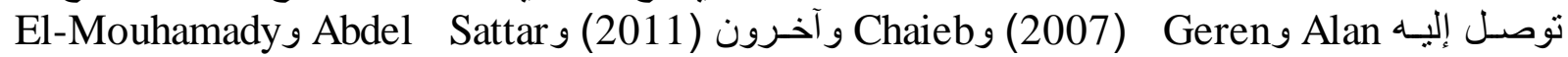

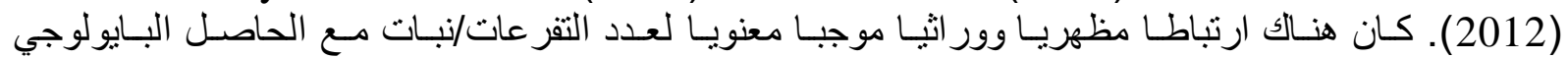

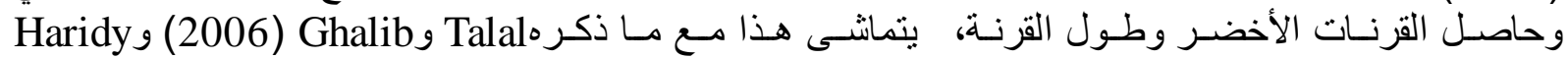

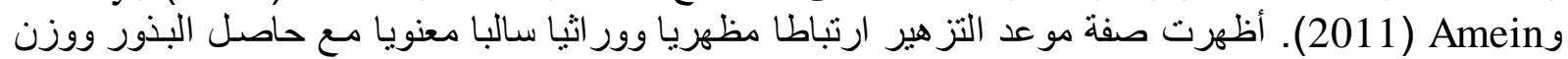

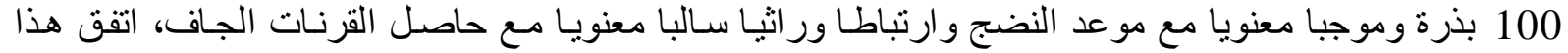

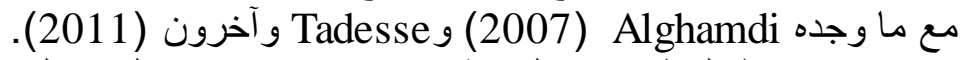

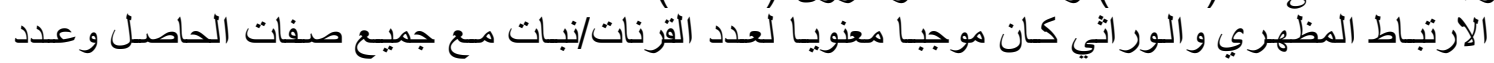

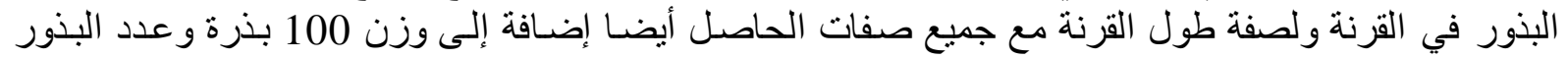

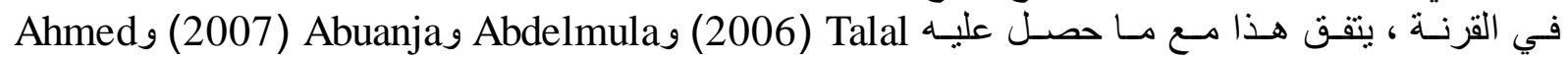

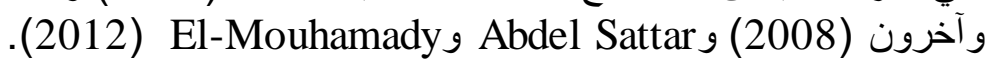


Mesopotamia J. of Agric.

Vol. (45) No. (4) 2017
ISSN: $2224-9796$ (Online)

ISSN: 1815 - $316 \mathrm{X}$ (Print)

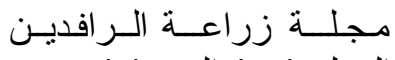

المجلد (45) العدد (4) 2017

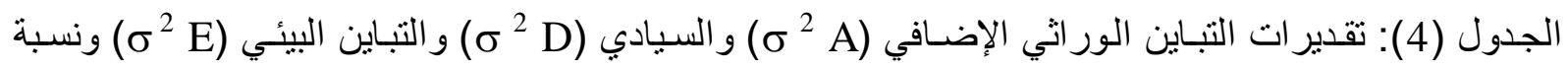
التوريث بالمعنيين الو اسع (

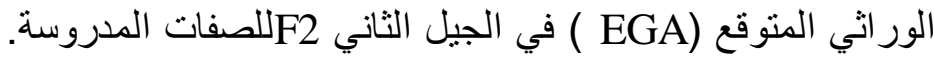

Table (4): Estimation of additive $\left(\sigma^{2} \mathrm{~A}\right)$, dominant $\left(\sigma^{2} \mathrm{D}\right)$ and environmental $\left(\sigma^{2} \mathrm{E}\right)$ variances, heritability in broad sense $\left(\% \boldsymbol{h}_{b . s}^{2}\right)$ and narrow sense $\left(\% \boldsymbol{h}_{n . s}^{2}\right)$ and average degree of dominance $(\bar{a})$ and genetic advance expectant (EGA) in $\mathrm{F}_{2}$ hybrids for studied characters.

\begin{tabular}{|c|c|c|c|c|c|c|}
\hline $\begin{array}{c}\text { طولة } \\
\text { القرنة } \\
\text { Pod } \\
\text { Pongth } \\
\text { lem) }\end{array}$ & $\begin{array}{c}\text { القرنات } \\
\text { عدات / } \\
\text { No. of } \\
\text { pods / } \\
\text { Plant }\end{array}$ & $\begin{array}{c}\text { مو النضج } \\
\text { (يوم) } \\
\text { Date } \\
\text { maturity } \\
\text { (days) }\end{array}$ & $\begin{array}{c}\text { التز هير } \\
\text { (يوم) } \\
\text { Date } \\
\text { Dlowering } \\
\text { flays) }\end{array}$ & 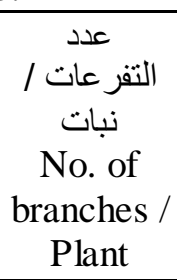 & $\begin{array}{c}\text { النبات } \\
\text { النفاع) } \\
\text { Plant } \\
\text { height } \\
\text { (cm) }\end{array}$ & $\begin{array}{l}\text { الثوابت الوراثية } \\
\text { Genetic } \\
\text { Parameters }\end{array}$ \\
\hline $\begin{array}{c}6.884 \\
\pm 4.390 \\
\end{array}$ & $\begin{array}{r}12.865 \\
\pm 9.852 \\
\end{array}$ & $\begin{array}{c}2.798 \\
\pm 1.849 \\
\end{array}$ & $\begin{array}{r}1.692 \\
\pm 1.725 \\
\end{array}$ & $\begin{array}{c}1.519 \\
\pm 1.044 \\
\end{array}$ & $\begin{array}{c}51.111 \\
\pm 34.996 \\
\end{array}$ & $\sigma^{2} \mathrm{~A}$ \\
\hline $\begin{array}{c}2.087 \\
\pm 2.215\end{array}$ & $\begin{array}{c}7.081 \\
\pm 14.908\end{array}$ & $\begin{array}{c}7.419 \\
\pm 7.674\end{array}$ & $\begin{array}{c}4.602 \\
\pm 6.834\end{array}$ & $\begin{array}{c}0.349 \\
\pm 0.772\end{array}$ & $\begin{array}{c}21.297 \\
\pm 33.098\end{array}$ & $\sigma^{2} \mathrm{D}$ \\
\hline $\begin{array}{c}0.229 \\
\pm 0.235 \\
\end{array}$ & $\begin{array}{c}9.077 \\
\pm 9.340 \\
\end{array}$ & $\begin{array}{c}0.478 \\
\pm 0.491 \\
\end{array}$ & $\begin{array}{r}2.995 \\
\pm 3.082 \\
\end{array}$ & $\begin{array}{c}0.483 \\
\pm 0.497 \\
\end{array}$ & $\begin{array}{r}15.456 \\
\pm 15.904 \\
\end{array}$ & $\sigma^{2} \mathrm{E}$ \\
\hline 97.508 & 68.725 & 95.530 & 67.756 & 79.453 & 82.408 & $\% h_{b . s}^{2}$ \\
\hline 74.817 & 44.328 & 26.161 & 18.215 & 64.588 & 58.169 & $\% h_{n . s}^{2}$ \\
\hline 0.778 & 1.049 & 2.302 & 2.332 & 0.678 & 0.912 & $\bar{a}$ \\
\hline 34.246 & 39.802 & 3.373 & 3.898 & 37.345 & 19.068 & EGA \\
\hline
\end{tabular}

\begin{tabular}{|c|c|c|c|c|c|c|}
\hline 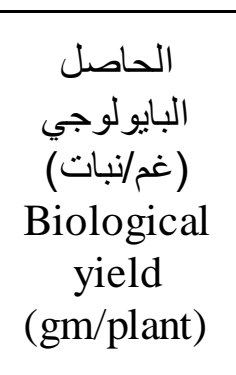 & $\begin{array}{c}\text { حاصل } \\
\text { البذور/تبات) } \\
\text { Seeds } \\
\text { yield } \\
\text { (gm/plant) }\end{array}$ & $\begin{array}{c}\text { القرنات } \\
\text { الجافل } \\
\text { القم/نبات) } \\
\text { Dry pods } \\
\text { yield } \\
\text { (gm/plant) }\end{array}$ & $\begin{array}{c}\text { القرنات الأخضر/ناتر) } \\
\text { (غرلبات } \\
\text { Green } \\
\text { pods yield } \\
\text { (gm/plant) }\end{array}$ & $\begin{array}{c}\text { وزن } 100 \\
\text { وزرة (غم) } \\
100 \text { seed } \\
\text { weight } \\
\text { (gm) }\end{array}$ & $\begin{array}{c}\text { البذور في } \\
\text { القرنة } \\
\text { No. of } \\
\text { seeds } \\
\text { per pod }\end{array}$ & $\begin{array}{c}\text { الثو ابثة } \\
\text { الور اثية } \\
\text { Genetic } \\
\text { Parameters }\end{array}$ \\
\hline $\begin{array}{c}21020.950 \\
\pm 15668.160\end{array}$ & $\begin{array}{c}309.442 \\
\pm 203.744\end{array}$ & $\begin{array}{c}489.336 \\
\pm 330.835\end{array}$ & $\begin{array}{c}8666.482 \\
\pm 5856.238\end{array}$ & $\begin{array}{c}242.581 \\
\pm 163.977\end{array}$ & $\begin{array}{c}0.250 \\
\pm 0.263\end{array}$ & $\sigma^{2} \mathrm{~A}$ \\
\hline $\begin{array}{c}11626.680 \\
\pm 22323.750\end{array}$ & $\begin{array}{c}81.050 \\
\pm 116.451\end{array}$ & $\begin{array}{c}137.462 \\
\pm 237.871\end{array}$ & $\begin{array}{c}3622.170 \\
\pm 5235.839\end{array}$ & $\begin{array}{c}15.245 \\
\pm 78.637\end{array}$ & $\begin{array}{c}0.167 \\
\pm 0.634\end{array}$ & $\sigma^{2} \mathrm{D}$ \\
\hline $\begin{array}{c}12798.030 \\
\pm 13169.060 \\
\end{array}$ & $\begin{array}{c}48.414 \\
\pm 49.818 \\
\end{array}$ & $\begin{array}{c}125.115 \\
\pm 128.742 \\
\end{array}$ & $\begin{array}{c}2198.977 \\
\pm 2262.729 \\
\end{array}$ & $\begin{array}{c}61.859 \\
\pm 63.652 \\
\end{array}$ & $\begin{array}{c}0.474 \\
\pm 0.488 \\
\end{array}$ & $\sigma^{2} \mathrm{E}$ \\
\hline 71.838 & 88.969 & 83.360 & 84.821 & 80.649 & 46.864 & $\% h_{b . s}^{2}$ \\
\hline 46.255 & 70.502 & 65.078 & 59.819 & 75.881 & 28.078 & $\% h_{n . s}^{2}$ \\
\hline 1.051 & 0.723 & 0.749 & 0.914 & 0.354 & 1.156 & $\bar{a}$ \\
\hline 40.689 & 59.274 & 54.520 & 53.595 & 19.516 & 17.189 & $\overline{\text { EGA }}$ \\
\hline
\end{tabular}


Mesopotamia J. of Agric.

Vol. (45) No. (4) 2017
ISSN: 2224 - 9796 (Online)

ISSN: 1815 - $316 \mathrm{X}$ (Print)

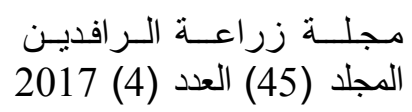

الجدول (5): معاملات الارتباط المظهري (القيم العليا) و الور اثي (القيم السفلى) بين الصفات المدروسة في الجيل الثاني F2 . . .

Table (5): Phenotypic ( up values) and Genotypic( under values) correlation for studied characters in $\mathrm{F}_{2}$ hybrids.

\begin{tabular}{|c|c|c|c|c|c|c|c|c|c|c|c|}
\hline $\begin{array}{c}\text { التفر عات/ عدات } \\
\text { عات } \\
\text { No. of } \\
\text { branches } \\
\text { / Plant }\end{array}$ & $\begin{array}{c}\text { التز هير } \\
\text { (يوم) } \\
\text { Date } \\
\text { flowering } \\
\text { (days) }\end{array}$ & $\begin{array}{c}\text { مو عد } \\
\text { لنضج) } \\
\text { Date } \\
\text { maturity } \\
\text { (days) }\end{array}$ & $\begin{array}{c}\text { القزنات/ عدات } \\
\text { البات } \\
\text { No. of } \\
\text { pods / } \\
\text { Plant }\end{array}$ & $\begin{array}{c}\text { طولة } \\
\text { القرنة } \\
\text { (س) } \\
\text { Pod } \\
\text { length } \\
\text { (cm) }\end{array}$ & $\begin{array}{c}\text { البذور فئة } \\
\text { القي } \\
\text { No. of } \\
\text { seeds } \\
\text { per pod }\end{array}$ & $\begin{array}{c}\text { وزبنّة } 100 \\
\text { وزذرة) } \\
100 \text { seed } \\
\text { weight } \\
\text { (gm) }\end{array}$ & $\begin{array}{c}\text { القرناتل } \\
\text { الأخضر/تبات) } \\
\text { Green pods } \\
\text { yield } \\
\text { (gm/plant) }\end{array}$ & 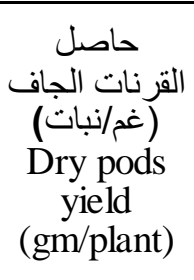 & 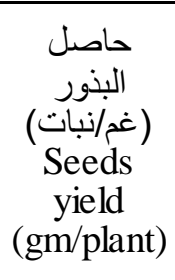 & $\begin{array}{c}\text { البايولوجئيل) } \\
\text { Biological } \\
\text { yield } \\
\text { (gm/plant) }\end{array}$ & $\begin{array}{c}\text { الصفات المدروسة } \\
\text { Studied Characters }\end{array}$ \\
\hline $0.434 *$ & $-0.443 *$ & $-0.578 * *$ & 0.310 & 0.262 & 0.183 & $0.522 * *$ & $0.686 * *$ & $0.673 * *$ & $0.641 * *$ & $0.683 * *$ & ارتفاع النبات(سم) \\
\hline \multirow[t]{20}{*}{$0.476 * *$} & $-0.467 * *$ & $-0.592 * *$ & 0.304 & 0.271 & 0.190 & $0.542 * *$ & $0.693 * *$ & $0.684 * *$ & $0.648 * *$ & $0.699 * *$ & Plant height $(\mathrm{cm})$ \\
\hline & -0.041 & -0.159 & 0.139 & $0.427 *$ & 0.199 & 0.046 & $0.415 *$ & 0.295 & 0.230 & $0.548 * *$ & عدد التفر عات/نبات \\
\hline & -0.040 & -0.161 & 0.148 & $0.433 *$ & 0.226 & 0.060 & $0.440 *$ & 0.316 & 0.252 & $0.568 * *$ & No. of branches/Plant \\
\hline & & $0.897 * *$ & -0.071 & 0.082 & -0.016 & $-0.371 *$ & -0.237 & -0.337 & $-0.403 *$ & -0.223 & مو عد التز هير(يوم) \\
\hline & & $0.916 * *$ & -0.075 & 0.088 & -0.001 & $-0.384 *$ & -0.262 & $-0.367 *$ & $-0.415 *$ & -0.255 & Date flowering (days) \\
\hline & & & -0.152 & 0.052 & 0.002 & -0.311 & -0.246 & -0.331 & -0.348 & -0.302 & موعد النضج(يوم) \\
\hline & & & -0.158 & 0.055 & 0.005 & -0.318 & -0.255 & -0.342 & -0.357 & -0.318 & Date maturity (days) \\
\hline & & & & 0.236 & $0.386 *$ & 0.166 & $0.446 *$ & $0.470 * *$ & $0.441 *$ & $0.512 * *$ & عدد القرنات/نبات \\
\hline & & & & 0.245 & $0.416 *$ & 0.173 & $0.453 *$ & $0.476 * *$ & $0.450 *$ & $0.523 * *$ & No. of pods / Plant \\
\hline & & & & & $0.734 * *$ & $0.396 *$ & $0.506 * *$ & $0.504 * *$ & $0.520 * *$ & $0.544 * *$ & طول القرنة (سم) \\
\hline & & & & & $0.778 * *$ & $0.407 *$ & $0.521 * *$ & $0.516 * *$ & $0.530 * *$ & $0.566 * *$ & Pod length $(\mathrm{cm})$ \\
\hline & & & & & & 0.297 & $0.380 *$ & $0.422 *$ & $0.457 *$ & $0.383 *$ & عدد البذور في القزنة \\
\hline & & & & & & 0.309 & $0.414 *$ & $0.456 *$ & $0.484 * *$ & $0.435 *$ & No. of seeds per pod \\
\hline & & & & & & & $0.559 * *$ & $0.652 * *$ & $0.723 * *$ & $0.386 *$ & وزن 100بذرة (غم) \\
\hline & & & & & & & $0.579 * *$ & $0.679 * *$ & $0.745 * *$ & $0.407 *$ & 100 seed weight (gm) \\
\hline & & & & & & & & $0.940 * *$ & $0.893 * *$ & $0.911 * *$ & حاصل القرنات الأخضر (غم/نبات) \\
\hline & & & & & & & & $0.952 * *$ & $0.909 * *$ & $0.925 * *$ & Green pods yield(gm/plant) \\
\hline & & & & & & & & & $0.954 * *$ & $0.857 * *$ & حاصل القرنات الجاف (غم/نبات) \\
\hline & & & & & & & & & $0.970 * *$ & $0.866 * *$ & Dry pods yield (gm/plant) \\
\hline & & & & & & & & & & $\begin{array}{l}0.774 * * \\
0.794 * *\end{array}$ & 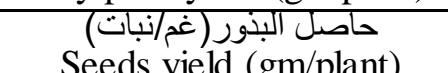 \\
\hline & & & & & & & & & & 0.134 & sce us yicia (gin/piam) \\
\hline
\end{tabular}


Mesopotamia J. of Agric.

Vol. (45) No. (4) 2017

كما وجد أن هناك ارتباطا مظهريا وور اثيا موجبا معنويا لصفتي عدد البذور في القرنة ووزن 100 بذرة

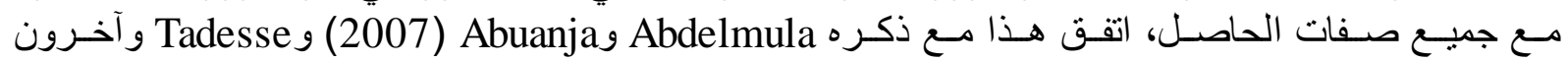

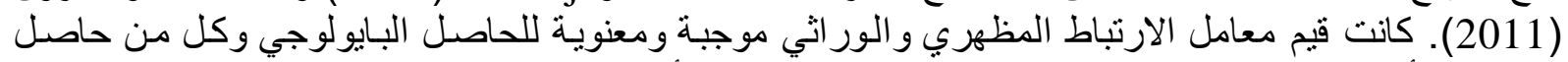

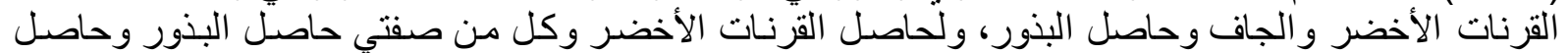

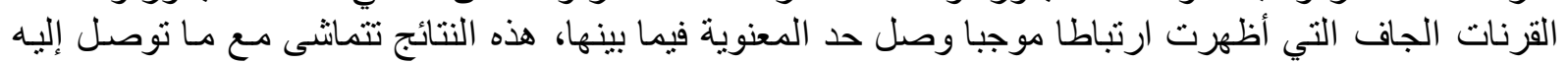

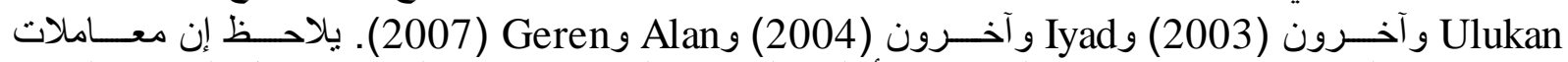

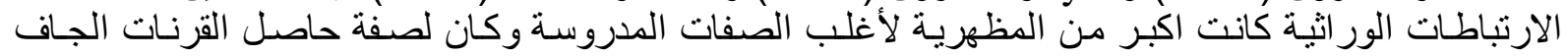

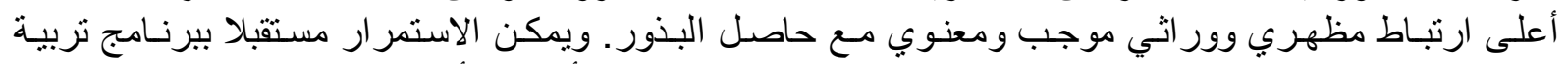

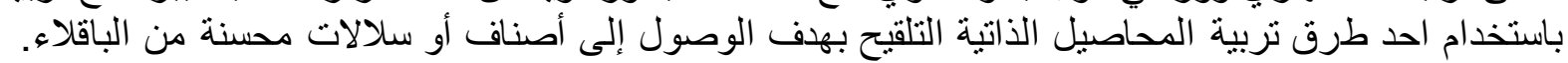
ومما سبق نستنتج ما يأتي: تانئ

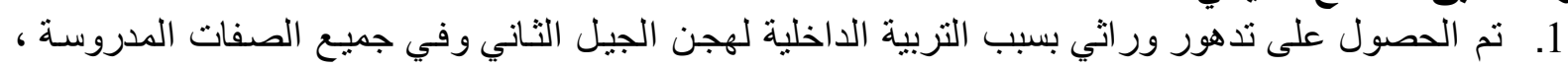

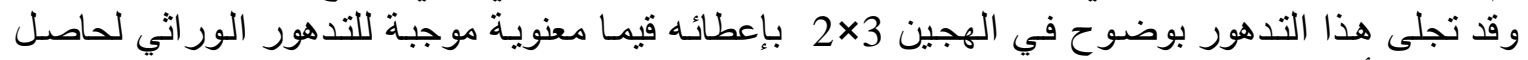

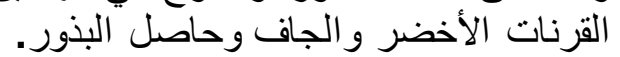

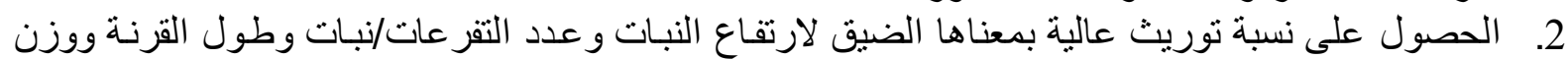

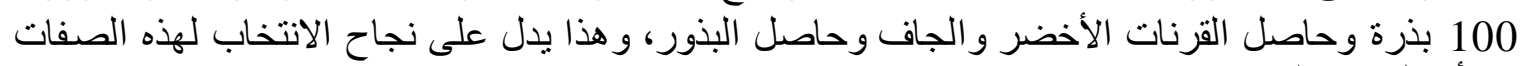
في أجيال انعز الية مبكرة

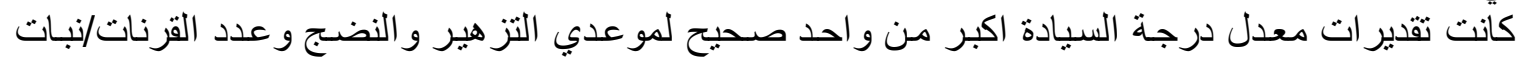

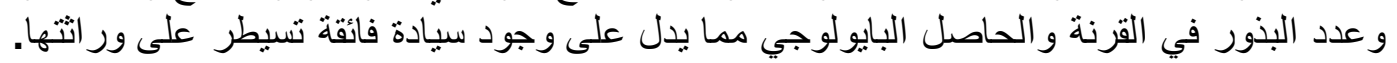

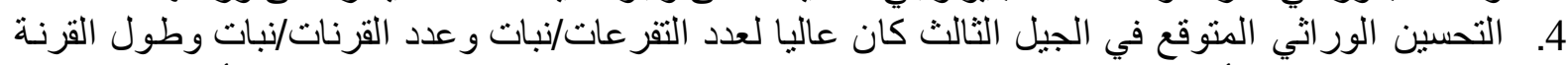

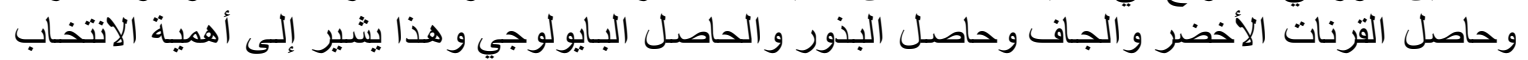

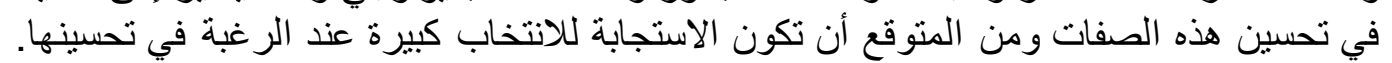

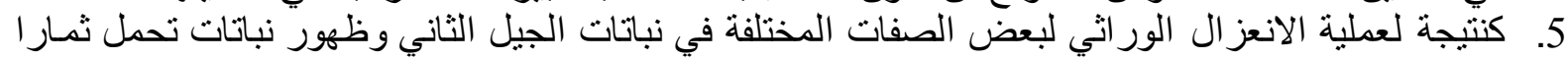

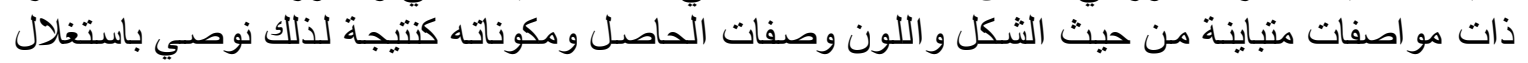
هذه الانعز الات في برات منباينة من التربية والتحسين.

\title{
INBREEDING DEPRESSION AND EXPECTANT GENETIC ADVANCE IN F2 HYBRIDS FABA BEAN (Vicia faba L)
}

\author{
Shamil Y.Hassan AL-Hamdany \\ Hort. \& Landscape Design Dept., College of Agriculture and Forestry, Mosul \\ University. Iraq \\ E-mail: Shamil1970@yahoo.com
}

\begin{abstract}
The study aimed to evaluate the performance of hybrids and their parents for identify promising hybrids and the objectives to develop single cross hybrids in addition to estimate inbreeding depression, some genetic parameters, phenotypic and genotypic correlation for the studied characters. An experiment was conducted by using four varieties of faba bean (Vicia faba L.) viz., 1- French (Aguadulce) 2- Syrian (Shami) 3- Spain and 4- Holland, were Full diallel crossing carried out according to (Griffing 1956) first method (Model I) to produce $F_{2}$-Hybrids of twelve single hybrid from self pollination of $F_{1}$ hybrid during the growing season 2009/2010. The parents and $\mathrm{F}_{2}$ hybrid were planted by using Randomized Complete Block Design (R.C.B.D.) with three replications at vegetable field of the Horticulture and landscape design department, College of Agriculture and Forestry, Mosul University, during the growing season 2010/2011.
\end{abstract}


Mesopotamia J. of Agric.

Vol. (45) No. (4) 2017
ISSN: 2224 - 9796 (Online)

ISSN: 1815 - $316 \mathrm{X}$ (Print)

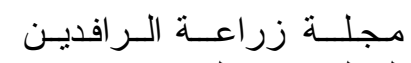

المجلد (45) العدد (4) 2017

Results showed that parents and $\mathrm{F}_{2 \mathrm{~S}}$ were significantly different for all the studied characters, parent 1- French (Aguadulce) was found to be the best for green, dry pods yield, seeds yield and biological yield, whereas the hybrid $(2 \times 3)$ for the green, dry pods yield and seeds yield, while the hybrid $(2 \times 1)$ for the biological yield. $\mathrm{F}_{2}$ hybrid exhibited a significant inbreeding depression for all the studied characters. A significant additive variance were found for all studied characters except date flowering and no.of seeds per pod. Narrow sense heritability was higher for: Plant height, no.of branches/plant, pod length, 100 seed weight, green, dry pods yield and seeds yield, which indicated an additive gene action for these characters. Over dominance were found for: flowering, maturity date, no.of pods/plant, no.of seeds per pod and biological yield. Genetic advance expectant in $\mathrm{F}_{3}$ hybrid were found for most studied characters, which indicates the importance of selection for improving these characters. The higher phenotypic and genotypic correlation were found between dry pods yield and seeds yield.

Keywords: Diallel Cross, Inbreeding Depression, Genetic Advance and Faba bean.

Received: 9/10/2013, Accepted: 2/12/2013.

\section{المصادر}

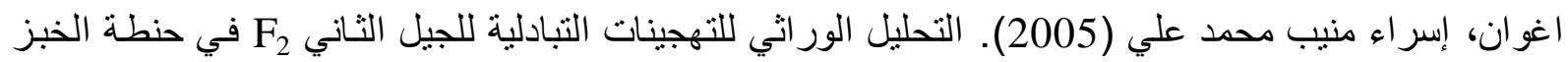

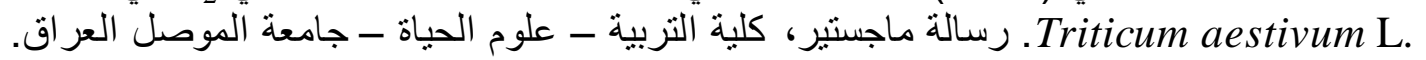

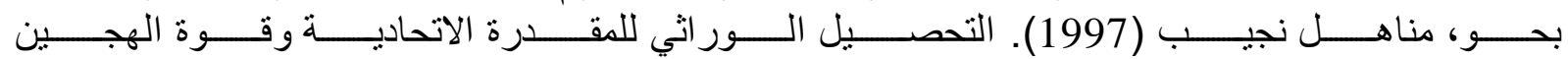
ومعامل المسار في الثعير.Hordeum vulgare L. أطروحة دكتور اه، قسم علوم الحياة- كلية العلوم -

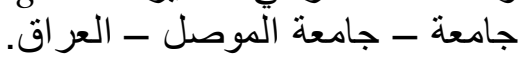

الحمداني، شامل يونس حسن (a) 2012). تقدير قوة الهجين و الفعل الجيني و الارتباط الور اثي و المظهري في

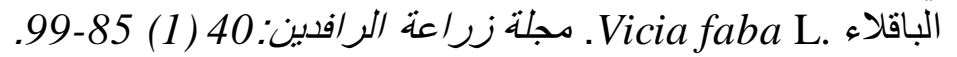

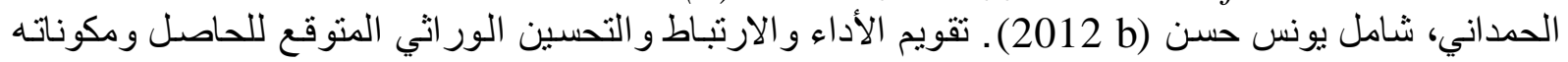

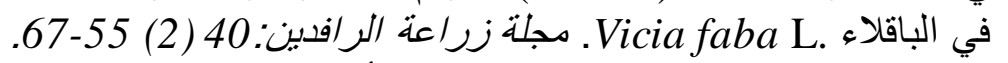

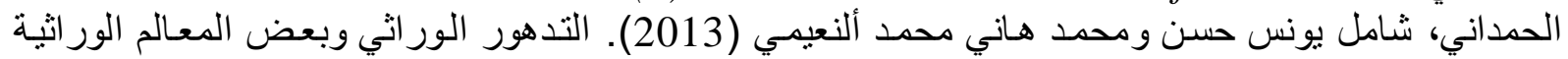

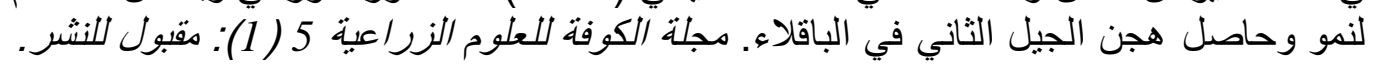

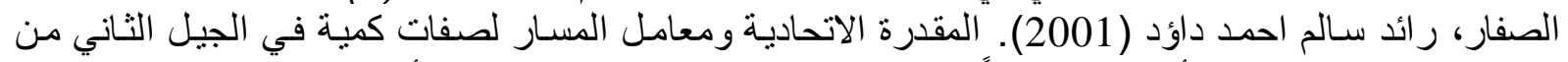

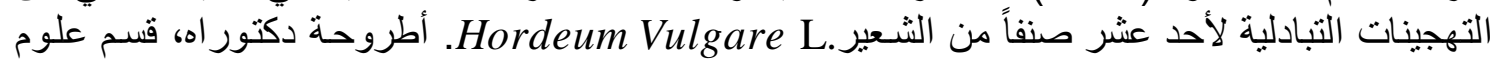

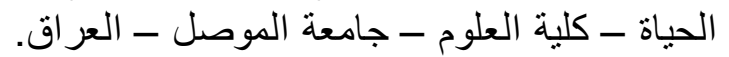

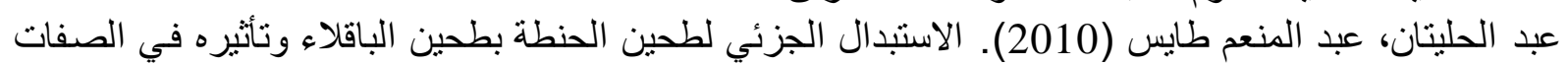

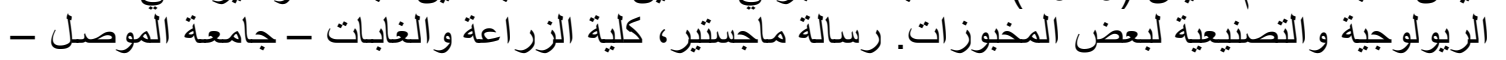
العر اق.

العذاري، عدنان حسن محمد (1999). أساسيات علم الور اثة. الطبعة الثالثة، مديرية دار الكتب للطباعة و النشر -

علي، عبده الكامل عبد الله .(1999). قوة الهجين و الفعل الجيني في الذرة الصفر اء .Zea mays Lلة أطروحة

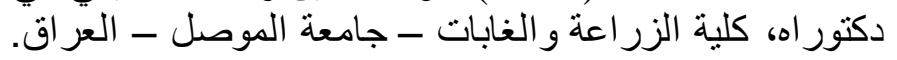

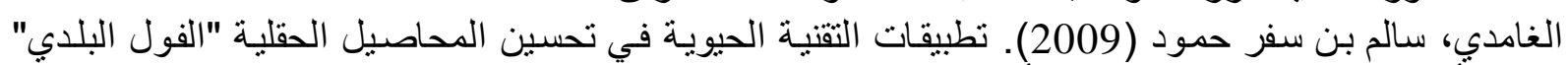

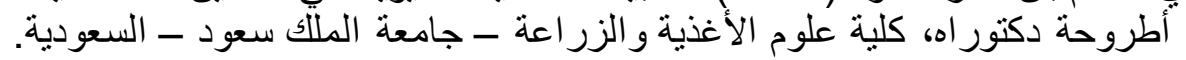

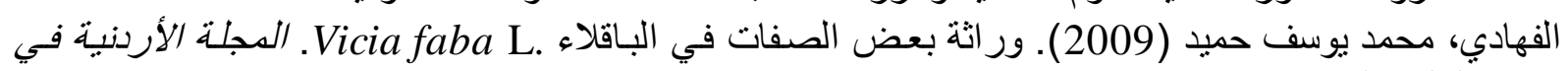

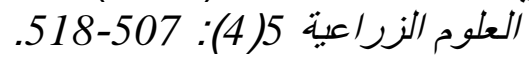

مجهول، (2010). الجهاز المركزي للإحصاء. إنتاج المحاصيل و الخضر اوات. مديرية الإحصاء الزر اعية هيئة

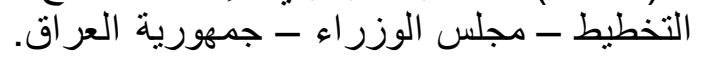

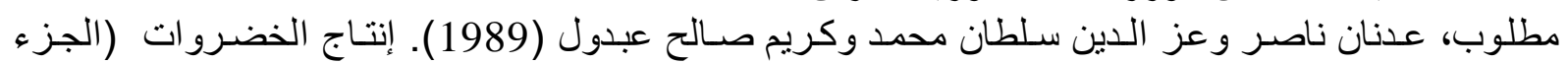

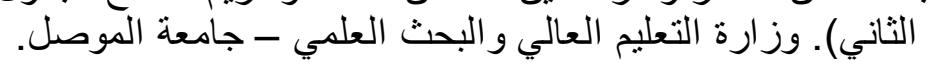

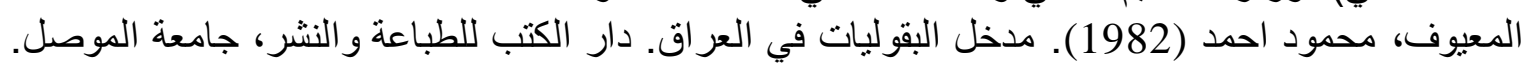




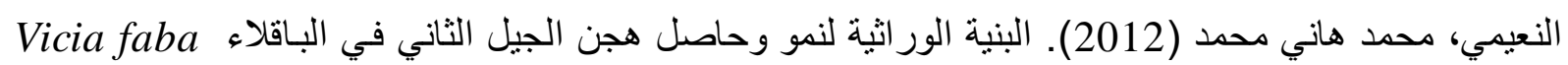

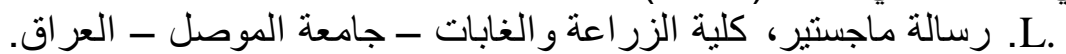

Abdalla, M.H. and A.M.A. Wahab (1995). Response of nitrogen fixation, nodule activities , and growth to potassium supply in water stressed broad bean. Journal of Plant Nutrition,18(7): 1391-1402.

Abdalla, M.M. ; D.S. Darwish ; M.M. El-Hady and E.H. El-Harty (2001). Investigations of faba beans Vicia faba L. $16 \mathrm{~F}_{1}$ and $\mathrm{F}_{2}$ diallel hybrids with reciprocals among five parents. Egypt Journal Plant Breeding, 5: 155-179.

Abdel Sattar, A.A. and A.A. El-Mouhamady (2012). Genetic analysis and molecular markers for yield and its components traits in faba bean Vicia Faba L.. Australian Journal of Basic and Applied Sciences, 6(7): 458-466.

Abdelmula, A.A. and I.K. Abuanja (2007). Genotypic responses, yield stability, and association between characters among some of Sudanese faba bean Vicia faba L. genotypes under Heat stress. Conference on International Agricultural Research for Development, October 9-11.

Ahmed, M.I (2005).Heterosis and inbreeding depression in faba bean Vicia faba L.. University of Khartoum Journal of Agricultural Science s, 13(2): 224-232.

Ahmed, M.S.H ; S.H.M. Abd El-Haleem ; M.A. Bakheit and S.M.S. Mohamed (2008). Comparison of three selection methods for yield and components of three faba bean Vicia faba L. crosses. World Journal of Agricultural Sciences, 4(5): 635639.

Alan, O. and H. Geren (2007). Evaluation of heritability and correlation for seed yield and its components in faba bean Vicia faba L.. Journal of Agronomy, 6(3): 484487.

Alghamdi, S.S. (2007). Genetic behavior of some selected faba bean genotypes. African Crop Sciences Conference Proceeding, 8: 709-714.

Alghamdi, S.S. (2008). Chemical composition of faba bean Vicia faba L. genotypes under various water regimes in Saudi Arabia. Green Farming, 1(8): 6-11.

Alghamdi, S.S. (2009). Heterosis and combining ability in diallel cross of eight faba bean Vicia faba L. genotypes. Asian Journal of Crop Sciences, 1(2): 66-76.

Ali, H.A.O. ; N.E.M. Mohamed ; A.A. Glala and M.H.Z. Eldekashy (2013). Heterosis and nature of gene action for yield and its components in faba bean Vicia faba L.. Journal of Plant Breeding and Crop Science, 5(3): 34-40.

Anonymous, (2002). Farm Chemicals Hand Book , (2002). III Meister Publishing Company. PP.828.

Chaieb, N. ; M. Bouslama and M. Mars (2011). Growth and yield parameters variability among faba bean Vicia faba L genotypes. Journal National Prod Plant resource., 1(2): 81-90.

Cochran, V.L. and S.F. Schlenther (1995). Intercropped oat and faba bean in Alaskadry matter production, dinitrogen fixation, nitrogen transfer and nitrogen fertilizer response. Agronomy, 87(3): 420-424.

Crepona, K. ; P. Marget ; C. Peyronnet ; B. Carrouéea ; P. Arese and G. Duc (2010). Nutritional value of faba bean Vicia faba L. seeds for feed and food. Field Crop Research,115: 329-339.

Duncan, D.B. (1955). Multiple Range and Multiple F-tests. Biometrics. 11: 1-42.

El-Bramawy, M.A.S. and M.A.M. Osman (2012). Diallel crosses of genetic enhancement for seed yield components and resistance to leaf miner and aphid 
infestations of Vici faba L.. International Journal of Agronomy and Agricultural Research (IJAAR), 2(2): 8-21.

El-Harty, E.H ; M. Shaaban ; M.M. Omran and S.B. Ragheb (2007). Heterosis and genetic analysis of yield and some characters in faba bean Vicia faba L.. Minia Journal of Agricultural Research \& Develop, 27(5): 897-913.

El-Sherbeeny, M. and L.D. Robertson (2006). Protein content variation in a pure line faba bean Vicia faba L. collection. Journal Science Food Agriculture, 58: 193196.

Farag, H.I.A. and S.A. Afiah (2012). Faba bean Vicia faba L. genotypes under Maryout conditions. Annals of Agricultural Science, 57(1): 37-46.

Farag, S.T. (2008). Hybridization effects on broad bean Vicia faba L. yield and its components. Minufiya Journal Agricultural Research, 33(2): 489-504.

Gasim, S. and W. Link (2007). Agronomic performance and the effect of selffertilization German winter faba bean. Journal of center European Agriculture, 8: $121-128$

Gnanasambandam, A. ; J. Paull ; A. Torres ; S. Kaur ; T. Leonforte ; H.Li ; X. Zong ; T. Yang and M. Materne (2012). Impact of molecular technologies on faba Bean Vicia faba L. breeding strategies. Agronomy, 2: 132-166.

Gomma, M.A.M and A.M.A. Shaheen (1995). Heterosis , Inbreeding depression , heritability and type of gene action in two intra barb a dense cotton crosses. Annals Agriculture sciences Ain Shams University, 40(1): 165-176.

Griffing, B. (1956). Concept of general and specific combining ability in relation to diallel crossing systems. Australian Journal Biological Sciences, 9: 463-493.

Gurmu, F. ; E. Lire1 ; A. Asfaw1 ; F. Alemayehu1 ; Y. Rezene and D. Ambachew (2012). Research article GGE-biplot analysis of grain yield of faba bean genotypes in southern Ethiopia. Electronic Journal of Plant Breeding, 3(3): 898907.

Haridy, A.G.H. and K.A. Amein (2011). The inheritance of some agronomical traits , protein content and seed beetle Callosobrucus maculates Fab. infestation in faba bean Vicia faba L.. Australian Journal of basic and applied sciences, 5(6): 1215 1222.

Hassan, E.E. (1997). Combining ability and factor analysis in durum wheat Triticum turgidum L.. Zigzag Journal Agricultural Research, 24(1): 23-36.

Ibrahim, H.M. (2010). Heterosis, combining ability and components of genetic variance in faba bean Vicia faba L.. Journal of King Abdulaziz University, Faculty of Meteorology, Environment and Arid Land Agriculture Sciences , 21(1): 35-50.

Iyad, W.M. ; J.H. Nizar ; M.T. Abdel-Rahman and O.S. Migdadl (2004). The importance of Bee - Pollination in four genotypes of faba bean Vicia faba L.. International Journal Agricultural, 6(1): 9-12.

Jensen, E.S. ; M.B. Peoples and H. Hauggaard-Nielsen (2010). Faba bean in cropping systems. Field Crops Research, 115: 203-216.

Kalia, P. ; S. Sood and Y. Sing (2003). Genetic variability in faba bean Vicia faba L. for pod yield and its contributing traits. Indian Journal Genetics, 63(3): 261-262.

Kalia, P. and S. Sood (2004). Genetic variation and association analyses for pod yield and other agronomic and quality characters an Indian Himalayan collection of 
broad bean Vicia faba L.. Sabrao Journal of Breeding and Genetics, 36(2): 55 61.

Kempthorne, B. (1969). An Introduction to Genetic Statistics. Ames Lows State Univ. press.

Kempthorne, O. (1957). An Introduction to Genetic Statistic. John Willey and Sons. New York.

Mather, K. and J.L. Jinks (1982). Introduction to Biometrical Genetics. Chapman and Hall Ltd. $3^{\text {rd }}$.

Rabie, E. ; M. Zakia ; K.A. Ezzat and A. Moniem (2003). Field components in faba bean. Egypt Journal applied Science, 19: 92-110.

Robinson, H.F. (1966). Quantitative genetics in relation to breeding on the centennial of mendelism. Indian Journal Genetics, 26 A: 171-187.

Tadesse, T. ; M. Fikere ; T. Legesse and A. Parven (2011). Correlation and path coefficient analysis of yield and its component in faba bean Vicia faba L. germplasm. International Journal of Biodiversity and conservation, 3(8): 376382 .

Talal, T. (2006). Impacts of row spacing on faba bean growth under Mediterranean rain fed conditions. Journal of agronomy, 5(3): 527-532.

Talal, T. and S. Ghalib (2006). Effect of planting date on faba bean Vicia faba L. nodulation and performance under semiarid conditions. World Journal of agriculture sciences, 2(4): 477-482.

Toker, C. (2004). Estimates of broad-sense heritability for seed yield and yield criteria in faba bean Vicia faba L.. Hereditas, 140: 222-225.

Ulukan, H. ; M. Guler and S. keskin (2003). A Path coefficient analysis of some yield and yield components in faba bean Vicia faba L. genotypes. Pakistan Journal Biological Sciences, 6: 1951-1955.

Walter, A.B. (1975). Manual of Quantitave Gentetics [ $3^{\text {rd }}$ edition], Washington State Univ. Press. U.S.A.

Welsh, J.R. (1981). Fundamentals of Plant Genetics and Breeding. John Wiley \&Sons, Inc. New York, USA. 